\title{
ON THE ASYMPTOTIC SOLUTIONS OF ORDINARY DIFFERENTIAL EQUATIONS, WITH AN APPLICATION TO THE BESSEL FUNCTIONS OF LARGE ORDER*
}

\author{
BY \\ RUDOLPH E. LANGER
}

1. Introduction. The investigations which have hitherto been made of the solutions of the ordinary linear differential equation

$$
u^{\prime \prime}(x)+p(x) u^{\prime}(x)+\left\{\rho^{2} \phi^{2}(x)+q(x)\right\} u(x)=0, \dagger
$$

with respect to their asymptotic dependence upon the complex parameter $\rho$, have almost without exception been restricted to the case in which $\phi^{2}(x)$, the coefficient of the parameter, remains positive (or negative) over the entire assigned interval of the variable $x$. The results in that case are familiar. $\ddagger \mathrm{A}$ determination of coefficients $\alpha_{i j}(x)$ in a pair of expressions of the type

$$
\begin{aligned}
& \phi^{-1 / 2}(x) e^{i \xi}\left\{1+\frac{\alpha_{11}(x)}{\rho}+\frac{\alpha_{12}(x)}{\rho^{2}}+\cdots\right\}, \\
& \phi^{-1 / 2}(x) e^{-i \xi}\left\{1+\frac{\alpha_{21}(x)}{\rho}+\frac{\alpha_{22}(x)}{\rho^{2}}+\cdots\right\}, \quad \xi=\rho \int_{x_{0}}^{x} \phi(x) d x,
\end{aligned}
$$

is possible, so that the resulting forms represent asymptotically a pair of solutions of the equation given. Moreover, each of these forms is then known to represent one and the same solution so long as $\rho$ remains in a region of the complex plane in which its pure imaginary part is either invariably greater than or invariably less than some constant.

The forms (2) are evidently of oscillatory or exponential type according as $\phi^{2}(x)$ is positive or negative on the $x$ interval under account, and on different intervals in which $\phi^{2}(x)$ is of opposite sign the forms are respectively of the two opposing types. Between two such intervals a change in the character of any solution must, therefore, take place. It is evident, however, that the manner of this change is not deducible from the forms (2), for whatever the magnitude of $\rho$ these forms fail to remain significant at a zero of $\phi^{2}(x)$.

* Presented to the Society, April 18, 1930; received by the editors April 11, 1930.

$\dagger \mathrm{It}$ is merely as a matter of convenience that $\rho^{2} \phi^{2}(x)$ is written rather than $\rho \phi(x)$.

$\ddagger$ Horn, Mathematische Annalen, vol. 52 (1899), pp. 340-362; Birkhoff, these Transactions vol. 9 (1908), p. 219. 
Hence there always exists some interval about such a zero in which the forms do not represent a solution. Because of this failure in the mode of representation, the relation between the solutions respectively represented by the forms (2) on opposite sides of a zero of $\phi^{2}(x)$ is obscured, and the law for its determination does not appear to have been given. Upon this law depends the complete determination of the form of any specific solution of the equation in different $x$ intervals.

Directly connected with this problem of the association of solutions represented by the forms (2) for different values of $x$, is that of the relation between the solutions so represented for any fixed value of $x$ but differently restricted values of $\rho$. Indeed, it will be shown that it is a single problem to determine completely the asymptotic forms in question for unrestricted values of both $x$ and $\rho$. It is this larger problem which is taken as the subject of the present discussion. It contains the more restricted problems already mentioned as integral parts, and may perhaps lay claim to some degree of interest and importance. Aside from its utility as illustrated by the application to be discussed below, it may be remarked, in particular, that a solution of the problem is a requisite to the general extension of the method of asymptotic forms to the treatment of such boundary problems as result when an equation of type (1) is subjected to boundary conditions which apply at points between which $\phi^{2}(x)$ becomes zero.

A special boundary problem of this latter type has on previous occasion* been considered by the author. While a solution of the general problem described above was not found necessary in that case, due to the special structure of the differential system considered, the discussion and results of that paper have been largely suggestive for the present considerations. In this connection it should be noted that the problem at hand as applied to a specialized equation of the type (1) was raised and briefly discussed by H. Jeffreys $\dagger$ in 1924 , in connection with an application to the solutions of the Mathieu equation. A resemblance of thought will be noticeable in a comparison of certain of the considerations of the present paper with those of the paper cited.

The procedure of the discussion may be briefly outlined as follows. It is shown to begin with, by the derivation of explicit formulas, that there may be associated with any given equation of the type (1) a certain "related" equation. This related equation on the one hand approximates the equation given in a specific sense and on the other hand is explicitly solvable with the use of Bessel functions of particular order and argument. It is shown then,

* These Transactions, vol. 31 (1930), pp. 1-24.

$\dagger$ Proceedings of the London Mathematical Society, (2), vol. 23 (1925), p. 428. 
at first for restricted values of $x$ and $\rho$, and subsequently for general values, that the solutions of the given equation are represented asymptotically by the known solutions of the related equation. The asymptotic form so obtained for any particular solution of the equation given is subject to discontinuous changes as $x$ passes from one to the other side of a zero of $\phi^{2}(x)$, or as the value of $\arg \rho$ passes certain specific bounds.* The law which governs this change is determined and is shown to depend upon the degree to which $\phi^{2}(x)$ vanishes at the zero under consideration. These deductions occupy Part I of the paper.

In Part II of the paper the theory of the preceding part is applied to a special equation which is known to be solved by the Bessel functions of order $\rho$ and argument $\rho e^{x}$. As a result the formulas for the functions $J_{\rho}, Y_{\rho}$ and $H_{\rho}$ with arguments $\rho \operatorname{sech} \alpha$ and $\rho \sec \beta$ are obtained for all real values of $\alpha$ and $\beta$ and for large positive values of $\rho$. These formulas are in the main those which are familiarly known as representing the functions. in question, and which are given, for instance, in Watson's treatise. For the so-called intermediate values of the arguments, however, the formulas here obtained show certain differences and possibly hold certain advantages over those heretofore given. In any event, it may be not without interest that the various formulas are derived here practically as a group and through the means of a special application of more or less general results, rather than, as has heretofore been the case, by methods which were especially developed and adapted to the peculiar ends in view and which vary considerably from one set of formulas to the next.

\section{PART I}

THE GENERAL THEORY OF THE ASYMPTOTIC FORMS

2. The given differential equation. A familiar change of dependent variable reduces the equation (1) to the normal form

$$
u^{\prime \prime}(x)+\left\{\rho^{2} \phi^{2}(x)-\chi(x)\right\} u(x)=0,
$$

in which $\chi(x)$ is a function simply determinable from the coefficients $p(x)$, and $q(x)$. This form of the equation is conveniently adapted to the considerations which are to be made. The transformation will, therefore, be supposed to have been carried out, and throughout the discussion the differential equation will be supposed given in the form (3).

The primary assumption which is to be made, and which principally

* It will be recalled that such changes of asymptotic form occur also in the theory of the Bessel functions, and that the phenomenon has been designated in that connection as the Stokes' phenomenon. 
characterizes the equations to which the discussion is devoted, is that the interval of the argument $x$ may include a zero of the coefficient $\phi^{2}(x)$. This and the remaining assumptions are made specific in the following statement of hypotheses.

(i) The function $\phi^{2}(x)$ vanishes at a point $x=x_{0}$ to the degree $\nu$, where $\nu$ is any real positive constant or zero.

(ii) On some interval $I_{1}$ which contains the point $x=x_{0}$, the function $\phi^{2}(x)$ has no zero other than that at $x_{0}{ }^{*}$

(iii) On some interval $I_{2}$ which contains the point $x=x_{0}$, the function $\left(x-x_{0}\right)^{-v} \phi^{2}(x)$ possesses a continuous second derivative and is real and positive except possibly for a constant complex factor.

(iv) The function $\chi(x)$ is defined on some interval $I_{3}$ which contains the point $x=x_{0}$, and is bounded on any finite portion of this interval.

In the case of any specifically given equation (3) the intervals $I_{1}, I_{2}$, and $I_{3}$, on which the respective hypotheses above are satisfied, may be finite or may extend to infinity in either or both directions. Inasmuch as all the hypotheses are fulfilled only for values of $x$ which are common to all three of these intervals, the variable $x$ will be taken throughout the following discussion to lie on an interval $I$, which is closed at such finite end points as it may have, which includes the point $x=x_{0}$, and which contains only points common to the three intervals $I_{1}, I_{2}$, and $I_{3}$. Subject to these specifications the sub-intervals into which $I$ is divided by the point $x=x_{0}$ may be either finite or infinite.

The hypotheses (i) to (iv) are concerned primarily with the character of the given equation in the proximity of the point $x=x_{0}$. In case the interval $I$ is infinite, it is to be expected that the character of the equation for values of $x$ remote from $x_{0}$ is likewise of significance. This is in fact so, and necessitates a further hypothesis which is to be found below in $\$ 7$.

Neither the form of the differential equation (3) nor the validity of the hypotheses made is affected either by the change of independent variable $x^{\prime}=x-x_{0}$, or by any transfer of a constant factor from the function $\phi^{2}(x)$ to the parameter $\rho^{2}$. Hence it may be assumed without loss of generality, firstly, that the origin is located at the zero of $\phi^{2}(x)$, i.e., that $x_{0}=0$, and, secondly, that the function $x^{-v} \phi^{2}(x)$ is real and positive.

Since the function $x^{-v} \phi^{2}(x)$ has by hypothesis a continuous second derivative, an application of Taylor's theorem yields the formula

$$
\phi^{2}(x)=x^{\nu}\left\{\alpha_{0}+\alpha_{1} x+\alpha_{2}(x) x^{2}\right\}
$$

* The case of several, or any number of isolated zeros of $\phi^{2}(x)$ would, of course, be treated by sub-dividing the interval and considering separately the sub-intervals containing just one zero. 
in which the coefficients are real, $\alpha_{0}$ and $\alpha_{1}$ being constants, and $\alpha_{2}(x)$ a function continuous on the interval $I$. For positive values of $x$ the function $\phi^{2}(x)$ is real and positive. For negative values of $x$ its specification is made definitive by the relation

$$
\arg \phi^{2}(x)=\arg x^{\nu}=\nu \pi .
$$

The function $\phi(x)$ will be defined as that root of $\phi^{2}(x)$ which is positive for positive values of $x$.

A material simplification of the manipulations which follow is attained by the use of suitable abbreviations, and by the introduction of certain functions determined by the equation at hand. To this end let the functions $\Phi(x), \Omega(x)$, and $\Psi(x)$ be defined respectively by the formulas

$$
\begin{aligned}
& \Phi(x)=\int_{0}^{x} \phi(x) d x, \\
& \Omega(x)=\frac{\Phi(x)}{\phi(x)}, \\
& \Psi(x)=\frac{\{\Phi(x)\}^{1 / 2-\mu}}{\{\phi(x)\}^{1 / 2}}
\end{aligned}
$$

in which the constant $\mu$ is given by the equation

$$
\mu=\frac{1}{\nu+2} .
$$

The further relations

$$
\xi=\rho \Phi(x), \tau=\rho \Phi(t)
$$

may be looked upon as defining the abbreviations $\xi$ and $\tau$.

It is a matter of simple verification that the functions

$$
x^{-1 /(2 \mu)} \Phi(x), x^{-1} \Omega(x), \Psi(x)
$$

are each real and positive, and have each on the interval $I$ a continuous second derivative. Their Taylor's developments lead, therefore, to the formulas

$$
\begin{array}{lll}
\Phi(x)=x^{1 /(2 \mu)}\left\{\beta_{0}+\beta_{1} x+\beta_{2}(x) x^{2}\right\}, & \beta_{0} \neq 0, \\
\Omega(x)=x\left\{\gamma_{0}+\gamma_{1} x+\gamma_{2}(x) x^{2}\right\}, & & \gamma_{0}=2 \mu, \\
\Psi(x)=\left\{\delta_{0}+\delta_{1} x+\delta_{2}(x) x^{2}\right\}, & & \delta_{0} \neq 0,
\end{array}
$$

in which the coefficients with subscript 2 are continuous functions, while those with subscripts 0 and 1 are constants easily computable from those in formula (4). The evaluation of $\gamma_{0}$ has been especially noted for a subsequent purpose. 
It is to be particularly remarked that the constant $\mu$ as given by formula (6) is in every.case less than or at most equal to $\frac{1}{2}$. This constant will occur prominently in many subsequent formulas.

3. The related differential equation. The relation

$$
y(x)=\rho^{k}\{\Omega(x)\}^{1 / 2} C(\xi)
$$

defines the function $y(x)$ in terms of a constant $k$ and a function $C$ which will for the moment be left unspecified. Upon suitable differentiation of this relation, and an application of the equality

$$
\Omega^{1 / 2} \phi^{\prime}+\frac{\Omega^{\prime} \phi}{\Omega^{1 / 2}}=\frac{\phi}{\Omega^{1 / 2}}
$$

which follows readily from the formulas (5), it is found that

$$
y^{\prime \prime}(x)=\left\{\frac{\Omega^{\prime \prime}}{2 \Omega}-\frac{\left(\Omega^{\prime}\right)^{2}}{4 \Omega^{2}}\right\} y(x)+\rho^{2+k} \phi^{2}(x) \Omega^{1 / 2}\left\{C^{\prime \prime}(\xi)+\frac{1}{\xi} C^{\prime}(\xi)\right\} .
$$

This result suggests the choice of the function $C$ as a cylinder function, for if $C$ is so chosen, say as a cylinder function of order $k$, then

$$
C^{\prime \prime}(\xi)+\frac{1}{\xi} C^{\prime}(\xi)=\left\{-1+\frac{k^{2}}{\xi^{2}}\right\} C(\xi),
$$

and the relation (9a) reduces in consequence to the form

$$
y^{\prime \prime}(x)+\rho^{2} \phi^{2} y(x)=\left[\frac{\left(\Omega^{2}\right)^{\prime \prime}-3\left(\Omega^{\prime}\right)^{2}+4 k^{2}}{4 \Omega^{2}}\right] y(x),
$$

namely to a differential equation for the function $y(x)$.

Superficially this equation (9b) is of the form of equation (3). However, since $\Omega(x)$ vanishes at $x=0$ a consideration of the coefficient enclosed within brackets in the right-hand member is not dispensable. This consideration is simply made. If the function within the brace of the second of formulas (5a) is denoted by the symbol $\Omega_{1}(x)$, i.e.,

$$
\Omega(x)=x \Omega_{1}(x),
$$

it is readily found that the coefficient in question may be written in the form

$$
\left[\frac{4 k^{2}-\left\{\Omega_{1}^{2}-x\left(\Omega_{1}^{2}\right)^{\prime}\right\}+x^{2}\left\{2 \Omega_{1} \Omega_{1}^{\prime \prime}-\Omega_{1}^{\prime 2}\right\}}{4 x^{2} \Omega_{1}^{2}}\right] \text {. }
$$

Now on the one hand $\Omega_{1}^{2}(x) \neq 0$, and on the other hand the function

$$
\Omega_{1}^{2}(x)-x\left\{\Omega_{1}^{2}(x)\right\}^{\prime}
$$


differs from the value $\Omega_{1}^{2}(0)$ by a quantity of which $x^{2}$ is a factor. It follows from this that the coefficient in question is continuous at $x=0$ if and only if $4 k^{2}-\Omega_{1}^{2}(0)=0$, i.e., from (5a) if $k^{2}=\mu^{2}$. The motivation for the choice of $C$ in formula (8) as a cylinder function of order $\pm \mu$, i.e., $C(\xi)=C_{ \pm \mu}(\xi)$, is clear. With this choice and with the choice $k=\mu$, the formula (8) may be written

$$
y(x)=\Psi(x) \xi^{\mu} C_{ \pm \mu}(\xi)
$$

the function $\Psi(x)$ here involved being that given in the third of formulas (5). By the deductions thus concluded the formula (10) solves explicitly the differential equation

$$
y^{\prime \prime}(x)+\left\{\rho^{2} \phi^{2}(x)-\omega(x)\right\} y(x)=0
$$

in which

$$
\omega(x)=\frac{1}{4 \Omega^{2}}\left\{\left(\Omega^{2}\right)^{\prime \prime}-3\left(\Omega^{\prime}\right)^{2}+4 \mu^{2}\right\} .
$$

In virtue of the continuity of $\omega(x)$ on the interval $I$, it is immediately evident that the differential equation (11) is possessed of every property hypothesized for the given equation (3). It is found convenient on this account to designate the equation (11) as related to the equation (3), or simply as the related equation. The result thus obtained is embodied in the following theorem:

Theorem 1. To every equation of type (3) which satisfies the hypotheses (i) to (iv), there corresponds a related equation (11) which is of the same type and involves the same coefficient $\phi^{2}(x)$, and which is explicilly solved by the formula (10).

4. The solutions of the related equation. By Theorem 1 a complete set of solutions of the related equation (11) may be obtained by substituting successively in formula (10) two linearly independent cylinder functions of order $\pm \mu$. As a first choice it is proposed to use for this purpose the Bessel functions of the third kind* with suitably chosen arguments. The choice of arguments is to be dictated by the desired asymptotic forms, and will depend, therefore, upon the ranges of the variables $x$ and $\rho$. The considerations involved are the following.

For values of the complex variable $z$ which are of large modulus, the Bessel functions $H_{\mu}^{(1)}(z)$ and $H_{\mu}^{(2)}(z)$ are of known asymptotic forms. These forms remain invariant, i.e., each of the functions is represented by one and the same corresponding analytic expression, so long as $z$ remains in a (any)

* Cf. G. N. Watson, A Treatise on the Theory of Bessel Functions, Cambridge University Press, 1922 , p. 73. This reference will be indicated in the text by [W]. 
specific right or left-hand half of the complex $z$ plane. However, if $z$ varies unrestrictedly the original expressions may cease to represent the functions in question, and the rôle may be filled in different half-planes by different analytic forms. This phenomenon will assume essential prominence in the later portions of the discussion. It is desirable at the moment, however, to evade the complications which it involves, and this may be simply done by a restriction of the variable $z$ to a specific half-plane. For the purpose immediately at hand, therefore, it is sufficient to note here the forms [W, p. 198]

$$
\begin{gathered}
H_{\mu}{ }^{(j)}(z) \sim A_{j}{ }^{\mu} z^{-1 / 2} e^{ \pm i z}\left[1+\sum_{m=1}^{\infty} \frac{(\mu, m)}{\left( \pm \frac{i}{2} z\right)^{m}}\right],(\mu, m) \text { constants } \\
A_{j}{ }^{\mu}=\left(\frac{2}{\pi}\right)^{1 / 2} e^{\mp(\mu+1 / 2) \pi i / 2},{ }^{*}
\end{gathered}
$$

valid for $-\pi / 2 \leqq \arg z \leqq \pi / 2$. These formulas will serve as a basis for the derivation of the asymptotic forms of those solutions of the related equation which are to be chosen.

The variable $x$ is real, but may be either positive or negative. The parameter $\rho$ and the quantity $\xi$ on the other hand are complex and may range over the entire respective complex planes. The manner and extent to which restrictions are imposed upon the locations of $x$ and $\rho$ by corresponding restrictions upon the location of $\xi$, and vice versa, is easily determined as follows.

Let the complex $\xi$ plane be divided into quadrants $\Xi_{k, l}, k=0, \pm 1$, $\pm 2, \cdots ; l=1,2$, by the relations

$$
\Xi_{k, 1}:\left(k-\frac{1}{2}\right) \pi<\arg \xi \leqq k \pi, \quad \Xi_{k, 2}: k \pi<\arg \xi \leqq\left(k+\frac{1}{2}\right) \pi .
$$

For $x>0$ the function $\Phi(x)$ is real and positive and therefore $\arg \xi=\arg \rho$. On the other hand, for $x<0$, arg $\Phi(x)=\pi /(2 \mu)$, and hence arg $\xi=\arg \rho$ $+\pi /(2 \mu)$. It is evident from this that

$\xi$ in $\Xi_{k, 1}$ corresponds to

$$
\left\{\begin{array}{l}
\text { either } x>0, \text { and }\left(k-\frac{1}{2}\right) \pi<\arg \rho \leqq k \pi, \\
\text { or } x<0 \text {, and }\left(k-\frac{1}{2}-\frac{1}{2 \mu}\right) \pi<\arg \rho \leqq\left(k-\frac{1}{2 \mu}\right) \pi ;
\end{array}\right.
$$

* The notation requires explanation. It will frequently be convenient as in the present case to write two formulas in one by the use of double signs together with an index $j$. It will be understood in every such case that the upper signs are to be associated with the value $j=1$ and the lower signs with $j=2$. 
$\xi$ in $\Xi_{k, 2}$ corresponds to

$$
\left\{\begin{array}{l}
\text { either } x>0, \text { and } k \pi<\arg \rho \leqq\left(k+\frac{1}{2}\right) \pi, \\
\text { or } x<0 \text {, and }\left(k-\frac{1}{2 \mu}\right) \pi<\arg \rho \leqq\left(k+\frac{1}{2}-\frac{1}{2 \mu}\right) \pi .
\end{array}\right.
$$

Any condition expressed in terms of $\xi$ and $x$ is, therefore, easily translated into terms of $\rho$ and $x$, and the mode of expression may be considered immaterial. The pair of adjacent quadrants $\Xi_{k, 1}$ and $\Xi_{k, 2}$ will be referred to as the half-plane $\boldsymbol{\Xi}_{k}$.

If $k$ is given any integral value, the formulas

$$
y_{k, j}(x)=\left\{\begin{array}{l}
\frac{\Psi(x)}{i^{k} A_{j}^{\mu}} \xi^{\mu} H_{\mu}^{(j)}\left(\xi e^{-k \pi i}\right), \text { if } k \text { is even, } \\
\frac{\Psi(x)}{i^{k} A_{3-j}^{\mu}} \xi^{\mu} H_{\mu}^{(3-j)}\left(\xi e^{-k \pi i}\right), \text { if } k \text { is odd, } j=1,2,
\end{array}\right.
$$

define, by Theorem 1, a pair of linearly independent solutions $y_{k, 1}(x), y_{k, 2}(x)$, for the equation (11). These solutions and their derivatives are of importance in the deductions which follow. The computation of the derivatives is simple. Thus, since [W, p. 74]

$$
\frac{d}{d z}\left\{z^{\mu} H_{\mu}^{(j)}(z)\right\}=e^{ \pm(1-\mu) \pi i} z^{\mu} H_{1-\mu}^{(j)}(z), j=1,2,
$$

while from formulas (7) and (5) it is found that

$$
\frac{d \xi}{d x}=\frac{\rho^{2 \mu} \xi^{1-2 \mu}}{\Psi^{2}(x)},
$$

it follows that

(18) $y_{k, j}(x)=\frac{\Psi^{\prime}(x)}{\Psi(x)} y_{k, j}(x)+\left\{\begin{array}{l}\frac{\rho^{2 \mu} e^{ \pm(1-\mu) \pi i}}{i^{k} \Psi(x) A_{j}^{\mu}} \xi^{1-\mu} H_{1-\mu}^{(j)}\left(\xi e^{-k \pi i}\right), \text { if } k \text { is even, } \\ \frac{\rho^{2 \mu} e^{\mp(1-\mu) \pi i}}{i^{k} \Psi(x) A_{3-i}^{\mu}} \xi^{1-\mu} H_{1-\mu}^{(3-j)}\left(\xi e^{-k \pi i}\right), \text { if } k \text { is odd }\end{array}\right.$

It is readily verified that the formulas (18) may be written in terms of the quantities

$$
\bar{y}_{k, j}(x)=\left\{y_{k, j}{ }^{\prime}(x)-\frac{\Psi^{\prime}(x)}{\Psi(x)} y_{k, j}(x)\right\} \frac{\Psi^{2}(x)}{i \rho^{2 \mu}}, \quad j=1,2,
$$


in the form

$$
\text { (16a) } \pm \bar{y}_{k, j}(x)=\left\{\begin{array}{l}
\frac{\Psi(x)}{i^{k} A_{j}^{1-\mu}} \xi^{1-\mu} H_{1-\mu}^{(j)}\left(\xi e^{-k \pi i}\right), \text { if } k \text { is even, } \\
\frac{\Psi(x)}{i^{k} A_{3-j}^{1-\mu}} \xi^{1-\mu} H_{1-\mu}^{(3-j)}\left(\xi e^{-k \pi i}\right), \text { if } k \text { is odd, } j=1,2
\end{array}\right.
$$

This suggests an advantage in the consideration of the functions (16a) in place of the derivatives (18), for on the one hand the values $y_{k, j}(x)$ are directly obtainable from the values $\bar{y}_{k, j}(x)$ and $y_{k, j}(x)$, while on the other hand the formulas (16a) are obtainable from the formulas (16) by the mere substitution of $1-\mu$ in place of $\mu$. Many of the following deductions concerned with the quantities (16) can, therefore, be adapted by slight and obvious changes to apply to the quantities (16a), and the pertinent results for the derivatives (18) may thus be cheaply obtained.

The asymptotic forms of the quantities (16) and (16a) for $|\xi|$ large and $\xi$ in the half-plane $\Xi_{k}$ may be found from the formulas (13). Thus when $\xi$ lies in $\Xi_{k}$ the value $\xi_{0}=\xi e^{-k \pi i}$ lies in the half-plane $\Xi_{0}$, namely, in the region of validity of the relations (13). The forms desired are, therefore, available in terms of $\xi_{0}$ and may, of course, be simply expressed in terms of $\xi$. It is thus found that

$$
\begin{aligned}
y_{k, j}(x) & \sim \Psi(x) \xi^{\mu-1 / 2} e^{ \pm i \xi}\left\{1+\sum_{m=1}^{\infty} \frac{c_{m}^{k, j}}{\xi^{m}}\right\}, \text { for } \xi \text { in } \Xi_{k}, \\
\pm \bar{y}_{k, j}(x) & \sim \Psi(x) \xi^{1 / 2-\mu} e^{ \pm i \xi}\left\{1+\sum_{m=1}^{\infty} \frac{\bar{c}_{m}^{k, j}}{\xi^{m}}\right\}, j=1,2 .
\end{aligned}
$$

The coefficients $c$ in these formulas are constants which are easily computed. A record of their explicit values is, however, not essential to the purpose at hand.

For subsequent use certain further facts concerning the quantities (16) and (16a) are to be noted. Inasmuch as all considerations are to be made on the assumption that $|\rho|$ is bounded from zero, any relation $|\xi|<N$, in which $N$ is a positive constant, restricts the values of $x$ to a finite portion of the interval $I$. For such values of $x$, however, the function $\Psi(x)$ is bounded, and since the quantity

$$
z^{q} H_{q}^{(j)}(z), \quad j=1,2 ; \quad q>0,
$$

remains bounded with $|z|$, and approaches a limit not zero as $z \rightarrow 0$, it follows that 


$$
\left|y_{k, j}(x)\right| \leqq M,\left|\bar{y}_{k, j}(x)\right| \leqq M, \text { for }|\xi| \leqq N, *
$$

and that the values $y_{k, j}(0)$ and $\bar{y}_{k, j}(0)$ are constants different from zero.

From the form of equation (11) it is observable that the Wronskian of the solutions $y_{k, j}(x)$ is independent of $x$. Its value, as computed from the formulas (16) and (17), is found to be

$$
W\left(y_{k, 1}, y_{k, 2}\right)=(-1)^{k} \frac{\rho^{2 \mu} \xi}{A_{1^{\mu} A_{2}{ }^{\mu}}} W\left(H_{\mu}^{(1)}\left(\xi e^{-k \pi i}\right), H_{\mu}^{(2)}\left(\xi e^{-k \pi i}\right)\right),
$$

and since the Wronskian on the right of this relation has the value [W, p. 76]

$$
-\frac{4 i}{\pi \xi e^{-k \pi i}}
$$

the formula reduces to

$$
W\left(y_{k, 1}, y_{k, 2}\right) \equiv-2 i \rho^{2 \mu} .
$$

For subsequent use it is desirable to note also the related formula

$$
D\left(y_{k, 1}, y_{k, 2}\right) \equiv\left|\begin{array}{ll}
y_{k, 1}(x) & y_{k, 2}(x) \\
\bar{y}_{k, 1}(x) & \bar{y}_{k, 2}(x)
\end{array}\right|=-2 \Psi^{2}(x)
$$

which follows directly from the equalities (19) and (22). The results of this section may be summarized as follows:

THEOREM 2. The formulas (16) define for the related differential equation (11) a pair of solutions $y_{k, 1}(x), y_{k, 2}(x)$, corresponding to any integer $k$. These solutions satisfy the relations (21) and (22), and are asymptotically given by the formulas (20) for the values of $x$ and $\rho$ which correspond to values of $\xi$ in the half-plane $\mathbf{\Xi}_{k}$.

5. The formal solution of the given equation. Let the given differential equation (3) be written in the form

$$
u^{\prime \prime}(x)+\left\{\rho^{2} \phi^{2}(x)-\omega(x)\right\} u(x)=\theta(x) u(x),
$$

in which

$$
\theta(x)=\chi(x)-\omega(x),
$$

and $\omega(x)$ is the function so designated in equation (11). The structure of the left members of equations (3a) and (11) is then the same, and familiar reasoning, in which the relation (3a) is looked upon as non-homogeneous, leads to the equation

\footnotetext{
* The symbols $M$ and $N$ are used here, and will be used in the work to follow, merely to designate "some positive constant." It is to be understood, therefore, that the constants so represented in different formulas are not necessarily the same.
} 


$$
u(x)=c_{1} y_{1}(x)+c_{2} y_{2}(x)-\int_{x_{0}}^{x} \frac{y_{1}(x) y_{2}(t)-y_{2}(x) y_{1}(t)}{W\left(y_{1}, y_{2}\right)} \theta(t) u(t) d t
$$

the values $c_{1}, c_{2}$, and $x_{0}$ being constants, and $y_{1}(x), y_{2}(x)$ any linearly independent solutions of equation (11). By actual substitution, it is easily verified that the equation (3a) is solved by any solution of the equation (24), howsoever the constants and solutions $y$ involved in the latter may be chosen. It is proposed to determine the form of certain solutions of equation (3a) through the means of establishing the existence and corresponding form of certain solutions of equation (24), with specific choices of the elements involved. These choices will be governed by the configuration of the values $x$ and $\rho$.

For any pair of values $x, \rho$, the corresponding value $\xi$ lies in some quadrant $\Xi_{k, l}$, and $\xi$ will remain in this quadrant if $x$ is restricted to maintain its sign and $\rho$ is confined to a corresponding quadrant of the $\rho$ plane as shown by the relations (15). It will be supposed that $x$ and $\rho$ are so restricted throughout the discussion immediately at hand. From the relations (15) it is clear that two distinct configurations of $x$ and $\rho$ correspond to $\xi$ in the quadrant $\Xi_{k, l}$. While a distinction between them is not necessary at this point, it will nevertheless be supposed that one and the same configuration persists throughout the discussion.

Under the assumption that $\xi$ is confined to the quadrant $\Xi_{k, l}$, the solutions $y_{k, j}(x), j=1,2$, are known to have the forms deduced for them in the preceding section. These solutions may in particular be chosen to serve as the functions $y$ involved in the formula (24). If the designation $u_{k, j}(x)$ is given to the solution $u$ corresponding to the choice of values $y_{1}=y_{k, 1}, y_{2}=y_{k, 2}$, $c_{j}=1, c_{3-j}=0$, the equation (24) becomes

$$
\begin{gathered}
\text { (24a) } u_{k, j}(x)=y_{k, j}(x)+\frac{1}{2 i_{\rho^{2 \mu}}} \int_{x_{0}}^{x}\left\{y_{k, 1}(x) y_{k, 2}(t)-y_{k, 2}(x) y_{k, 1}(t)\right\} \theta(t) u_{k, j}(t) d t \\
j=1,2,
\end{gathered}
$$

A determination of the constant $x_{0}$ remains to be made, but will for the moment be deferred.

Let the quantities $U_{j}(x)$ and $Y_{j}(x)$ be defined by the formulas

$$
U_{j}(x)=\frac{u_{k, j}(x)}{\Psi(x)} e^{\mp i \xi}, \quad Y_{j}(x)=\frac{y_{k, j}(x)}{\Psi(x)} e^{\mp i \xi}, j=1,2 .
$$

With the use of these functions the equation (24a) may be written in the form 


$$
U_{j}(x)=Y_{j}(x)+\frac{1}{\rho^{2 \mu}} \int_{x_{0}}^{x} K_{j}(x, t, \rho) U_{j}(t) d t, \quad j=1,2,
$$

with

$$
K_{j}(x, t, \rho)= \pm \frac{\theta(t) \Psi^{2}(t)}{2 i}\left\{Y_{j}(x) Y_{3-j}(t)-Y_{3-j}(x) Y_{j}(t) e^{\mp 2 i(\xi-r)}\right\} .
$$

It will be recalled that the symbol $\tau$ here involved was defined in formulas (7).

The equation (24b) is in form an integral equation for $U_{j}(x)$, and as such may be iterated in familiar fashion, the quantity $U_{i}$ under the sign of integration being replaced by the entire right-hand member of the equatio ${ }^{*}$ A continued repetition of this process leads ultimately to the formal equality

in which

$$
U_{j}(x)=Y_{j}(x)+\sum_{n=1}^{\infty} \frac{Y_{j}^{(n)}(x)}{\rho^{n \sigma}}
$$

$$
\begin{aligned}
Y_{j}^{(n)}(x) & =\rho^{\sigma-2 \mu} \int_{x_{0}}^{x} K_{j}(x, t, \rho) Y_{j}^{(n-1)}(t) d t \quad(n=1,2, \cdots ; j=1,2), \\
Y_{j}^{(0)}(x) & =Y_{j}(x) .
\end{aligned}
$$

The relations (27) and (28) when taken together are independent of the constant $\sigma$ which has been introduced into them. It will be seen later that a specific choice of this constant is a source of convenience for the discussion.

The infinite series on the right of the relation (27) formally satisfies the equation (24b). It will be shown in the following sections that a choice of the undetermined elements involved may be made so that the series converges uniformly and the relation (27) in consequence represents a true solution.

Let the functions $\bar{u}_{k, i}(x)$ be defined in a manner analogous to (19) by the formula

$$
\bar{u}_{k, j}(x)=\left(u_{k, j}^{\prime}(x)-\frac{\Psi^{\prime}(x)}{\Psi(x)} u_{k, j}(x)\right) \frac{\Psi^{2}(x)}{i^{2 \mu}}, \quad j=1,2 .
$$

If it is observed that the equation (24a) may be formally differentiated by the mere substitution of $u_{k, j}{ }^{\prime}$ and $y_{k, j}{ }^{\prime}$ respectively for the quantities $u_{k, j}$ and $y_{k, j}$ where they occur with the argument $x$, then it is readily seen also that the similar substitution of $\bar{u}_{k, j}(x)$ and $\bar{y}_{k, j}(x)$ for $u_{k, j}(x)$ and $y_{k, j}(x)$ in (24a) leads to a valid formula. It follows that the formal considerations made above for the functions $u_{k, j}(x)$ may be made equally well and with similar result for the functions $\bar{u}_{h, i}(x)$. 
6. Lemmas. The proof of the existence of a set of solutions $U_{j}(x)$ of equation (24b), and the associated investigation of their structure, is to be based upon the relation (27) obtained formally in $\$ 5$. It is requisite to this end that the infinite series involved in the relation be shown convergent. This is conveniently done by means of the facts which are framed below in the form of a set of simple lemmas.

Let $N$ be an arbitrarily chosen positive constant and consider the relation

$$
|\rho \Phi(\bar{x})|=N \text {. }
$$

The function $\Phi(x)$ is continuous on the interval $I$. From its definition it is clear, moreover, that $|\Phi(x)|$ increases monotonically with $|x|$ on each of the sub-intervals into which $I$ is divided by the point $x=0$. If $I^{*}$ is used to designate that one of these sub-intervals which contains $x$ under the configuration of values which was assumed in the preceding section, it follows that a unique point $\bar{x}$ on the interval $I^{*}$ is determined by the relation (30) for each value of $\rho$. The dependence of $\bar{x}$ upon $\rho$ is evident. In virtue of the formulas $(5 \mathrm{a})$ it is seen that this dependence satisfies quantitatively a relation of the type

$$
m_{1}|\rho|^{-2 \mu} \leqq|\bar{x}| \leqq m_{2}|\rho|^{-2 \mu},
$$

in which $m_{1}$ and $m_{2}$ are suitably determined positive constants.

The lemmas which follow are concerned with the evaluation of an integral

$$
I(\alpha, \beta)=\int_{\alpha}^{\beta} H(x, t, \rho) d t,
$$

subject to the following specifications:

(a) The points $\alpha$ and $\beta$ lie on the interval $I^{*}$;

(b) The configuration of values $x, \rho$ is that assumed in $\$ 5$;

(c) The integrand satisfies a pair of relations

$$
\begin{gathered}
|H(x, t, \rho)|<M h(t), \text { for } t \text { on } I^{*} \text { and }|\tau| \leqq N, \\
\left|\tau^{\delta} H(x, t, \rho)\right|<M h(t), \text { for } t \text { on } I^{*} \text { and }|\tau|>N,
\end{gathered}
$$

in which the exponent $\delta$ is a specific positive constant, and $h(t)$ is a function continuous on the interval $I^{*}$.

Lемма 1. If $0 \leqq|\alpha| \leqq|\beta| \leqq|\bar{x}|$, then

$$
|I(\alpha, \beta)| \leqq M_{1}|\rho|^{-2 \mu} \text {. }
$$

The proof is simple. On the interval of integration the first of relations (33) is satisfied. Since $h(t)$ is bounded, the same is true of the entire inte- 
grand, and by relation (31) the length of the interval of integration is of the order of $|\rho|^{-2 \mu}$.

LemMa 2. If $x_{c}$ is any fixed point (i.e., independent of $\rho$ ) on the interval $I^{*}$, and if $|\bar{x}| \leqq|\alpha| \leqq|\beta| \leqq\left|x_{c}\right|$, then

$$
|I(\alpha, \beta)| \leqq \begin{cases}M_{2}|\rho|^{-\delta}, & \text { if } \mu>\delta / 2 \\ M_{2}|\rho|^{-\delta} \log |\rho|, & \text { if } \mu=\delta / 2 \\ M_{2}|\rho|^{-2 \mu}, & \text { if } \mu<\delta / 2\end{cases}
$$

On the interval of integration the second of formulas (33) is valid, and inasmuch as this interval is finite the function $h(t)$ is bounded. It is clear, therefore, that the integrand is of the order of $\tau^{-\delta}$. Since by formula (5a) $\tau$ is of the order of $\rho t^{1 /(2 \mu)}$, it follows that

$$
|I(\alpha, \beta)| \leqq M|\rho|^{-\delta} \int_{|\bar{x}|}^{\left|x_{c}\right|} \frac{d t}{t^{\delta /(2 \mu)}} .
$$

The conclusion is at hand because of the relation (31).

If the interval $I^{*}$ is finite, the discussion of cases arising from possible choices of $\alpha$ and $\beta$ is completely covered by the Lemmas 1 and 2, for the point $x_{c}$ may in particular be chosen as the end point of $I^{*}$. If the interval extends to infinity, however, a complete discussion must include also the further lemma which follows.

LEммA 3. If $\left|x_{c}\right| \leqq|\alpha| \leqq|\beta| \leqq \infty$ and if the integral

$$
\int_{x_{c}}^{\infty} \frac{h(t)}{\{\phi(t)\}^{\delta}} d t
$$

extended over the interval $I^{*}$, is convergent, then

$$
|I(\alpha, \beta)| \leqq M_{3}|\rho|^{-\delta}
$$

From the second of relations (33) the integrand of $I(\alpha, \beta)$ is of the order of $h(t) / \tau^{\delta}$. Since this quantity is positive on the interval $I^{*}$, except possibly for a constant complex factor, the substitution of the value of $\tau$ from formula (7) yields the relation

$$
|I(\alpha, \beta)| \leqq M|\rho|^{-\delta}\left|\int_{\alpha}^{\beta} \frac{h(t)}{\{\Phi(t)\}^{\delta}} d t\right| .
$$

The conclusion follows.

7. The dominant solution of the given equation for $\xi$ in a quadrant $\boldsymbol{\Xi}_{k, l}$. From the definition of the quadrants $\Xi_{k, l}$ as given in the relations (14), it is 
directly evident that when $\xi$ remains on such a quadrant the real part of $i \xi$ is either always positive oral ways negative. Hence one and the same one of the two solutions $y_{k, j}(x)$ remains dominant for all such values of $\xi$. Whether the subscript value associated with this dominant solution is $j=1$, or $j=2$, will depend, of course, upon the particular quadrant $\boldsymbol{\Xi}_{k, \iota}$ under consideration, namely, upon the values $k$ and $l$. To avoid an unessential differentiation of cases the value $j$ in question will be designated simply by $j=j^{\prime}$.

The subject of immediate attention in the present section is the derivation of a solution of the equation (24b) from the relation (27) for the value $j=j^{\prime}$. This involves a determination of the conditions under which the relation is convergent, and centers, therefore, upon a consideration of the quantities $Y_{j^{\prime}}^{(n)}(x)$ defined by the formula (28).

The constant $x_{0}$ was introduced into the formulas of $\S 5$, but was referred for later specification. This specification for the case in hand will now be made as follows, namely, when $j=j^{\prime}$, then $x_{0}=0$. It will be understood throughout this section that this value of $x_{0}$ has been fixed upon, and it will be understood likewise without repeated specific mention of the fact that $j$ temporarily takes the single value $j^{\prime}$ in the various formulas to be written.

The definitions (25) together with the formulas (20) and (21) yield readily the inequalities

$$
\begin{array}{r}
\left|Y_{j}(x)\right|<M, \text { for }|\xi| \leqq N, \\
\left|\xi^{1 / 2-\mu} Y_{j}(x)\right|<M, \text { for }|\xi|>N .
\end{array}
$$

These two relations may be combined into the single one

$$
\left|\xi^{(1 / 2-\mu) \cdot} Y_{j}(x)\right|<M
$$

if it is agreed to assign to the symbol $s$ the value

$$
s=\left\{\begin{array}{l}
0, \text { if }|\xi| \leqq N, \\
1, \text { if }|\xi|>N .
\end{array}\right.
$$

This agreement of notation will be adopted and the symbol $s$ will be used in accordance with it whenever a multiplicity of written formulas can thereby be avoided. For the sake of clarity the use of the letter $s$ in any sense other than that defined by the relation (35) will be avoided.

The functions $Y_{j}^{(n)}(x)$ depend by the relations (28) upon the kernel $K_{j}(x, t, \rho)$ which is defined in the formula (26). This formula involves on the one hand the functions $Y_{1}$ and $Y_{2}$ which are bounded in virtue of the relations (34), and on the other hand the factor $\exp \{\mp 2 i(\xi-\tau)\}$ which is now to be considered. As a result of the choice of $x_{0}$ the variable of integration, $t$, in formulas (24b) and (28) ranges on the interval $I^{*}$ between 0 and $x$. Clearly 
then $|\tau| \leqq|\xi|$. Since the value $\arg t$ is constant on the interval $I^{*}$ it follows that for the values of $t$ in hand

$$
\arg [i(\xi-\tau)]=\arg i \xi .
$$

The exponent $\mp 2 i(\xi-\tau)$ in formula (26) is, therefore, of real part opposite in sign to that of the corresponding exponent in the formula (20). Inasmuch as the latter is positive for $j=j^{\prime}$, in virtue of the determination of $j^{\prime}$, the exponential in $K_{j}(x, t, \rho)$ is bounded when $|t|$ does not exceed $|x|$. This conclusion, together with the inequalities (34), establishes, for $|t| \leqq|x|$, the relations

$$
\begin{array}{r}
\left|\xi^{(1 / 2-\mu)} K_{j}(x, t, \rho)\right| \leqq M \Psi^{2}(t)|\theta(t)|, \text { for }|\tau| \leqq N \\
\left|\tau^{(1 / 2-\mu)} \xi^{(1 / 2-\mu) s} K_{j}(x, t, \rho)\right| \leqq M \Psi^{2}(t)|\theta(t)|, \text { for }|\tau|>N .
\end{array}
$$

It is proposed to utilize this result to show that the quantities $Y_{i}^{(n)}(x)$ of formula (28) will satisfy the inequalities

$$
\left|\xi^{(1 / 2-\mu) s} Y_{j}^{(n)}(x)\right| \leqq M^{n+1} \quad(n=0,1,2, \cdots),
$$

provided firstly that the positive constant $M$ is suitably determined, and secondly that the hitherto unspecified constant $\sigma$ in formulas (28) is properly chosen.

The relation (37) is valid for $n=0$ since it reduces for that value to the relation (34). The proof of the general validity of (37) by induction will accordingly be complete if it is shown that the assumption of its validity with $n$ replaced by $(n-1)$ is sufficient to establish it as written. This may be done as follows.

If in (37) the value $(n-1)$ is substituted for $n$ and $t$ is written in place of $x$, the hypothesis tentatively adopted becomes

$$
\begin{aligned}
\left|Y_{j}^{(n-1)}(t) M^{-n}\right| & <1, \text { when }|\tau| \leqq N, \\
\left|\tau^{1 / 2-\mu} Y_{j}^{(n-1)}(t) M^{-n}\right| & <1 \text {, when }|\tau|>N .
\end{aligned}
$$

These inequalities together with the relations (36) show that the function

$$
\xi^{(1 / 2-\mu) s} K_{j}(x, t, \rho) Y_{j}^{(n-1)}(t) M^{-n}
$$

satisfies, with the value $\delta=1-2 \mu$, the hypotheses imposed in $\S 6$ upon the function $H(x, t, \rho)$. This is true, moreover, independently of the value assumed for $n$. Hence the formula (28) may be expressed in terms of integrals with the structure of those discussed in the lemmas of $\$ 6$. The attendant considerations depend upon the range of values ascribed to the variable $x$ and are the following. 
Case 1. $|x| \leqq|\bar{x}|$. In this case $|\xi| \leqq N$. The function (38) with $s=0$ may be designated, therefore, by $H(x, t, \rho)$, and as a result the formula (28) becomes

$$
Y_{j}^{(n)}(x)=M^{n} \rho^{\sigma-2 \mu} I(0, x) .
$$

Lemma 1 is applicable to the right member of this equation and yields the inequality

$$
\left|Y_{j}^{(n)}(x)\right| \leqq M^{n}|\rho|^{\sigma-4 \mu} M_{1}
$$

in which the value $M_{1}$ is independent of $n$ since the hypotheses on $H(x, t, \rho)$ are satisfied by the function (38) uniformly with respect to $n$. If $M$ is chosen at least as great as the specifiable value $M_{1}$, the relation (37) follows for the values of $x$ momentarily under consideration, provided $\sigma$ is chosen not to exceed the value $4 \mu$.

Case 2. $|\bar{x}|<|x| \leqq\left|x_{c}\right|$. In this case $|\xi|>N$, and the rôle of the function $H(x, t, \rho)$ may be assumed by the function (38) with $s=1$. Hence the formula (28) is expressible, after multiplication by $\xi^{1 / 2-\mu}$, in the form

$$
\xi^{1 / 2-\mu} Y_{j}^{(n)}(x)=M^{n} \rho^{\sigma-2 \mu}\{I(0, \bar{x})+I(\bar{x}, x)\} .
$$

Lemmas 1 and 2 are applicable to the respective terms on the right of this relation, and serve to establish the inequality

$$
\left|\xi^{1 / 2-\mu} Y_{j}^{(n)}(x)\right| \leqq M^{n}\left\{M_{1}|\rho|^{\sigma-4 \mu}+M_{2}|\rho|^{\sigma-\sigma_{1}}\right\},
$$

the constant $\sigma_{1}$ which is involved being defined by the appropriate formula

$$
\sigma_{1}=\left\{\begin{array}{l}
1, \quad \text { if } \mu>\frac{1}{4}, \\
1-\epsilon, \text { with } \epsilon>0 \text { but arbitrarily small, if } \mu=\frac{1}{4}, \\
4 \mu, \text { if } \mu<\frac{1}{4} .
\end{array}\right.
$$

Since $M$ may be chosen to exceed both $M_{1}$ and $M_{2}$, it is clear that the relation (37) will follow in this case also provided $\sigma$ is chosen not to exceed $\sigma_{1}$.

If the interval $I^{*}$ is finite, the point $x_{c}$ in Case 2 may in particular be chosen as the end point of this interval. The cases considered then exhaust the discussion. In the case of an infinite interval $I^{*}$, however, the discussion must be extended by a consideration also of the following additional case.

Case 3. $\left|x_{c}\right|<|x|$. The procedure of Case 2 is applicable without change to the case in hand, and by means of it the formula

$$
\xi^{1 / 2-\mu} Y_{j}^{(n)}(x)=M^{n} \rho^{\sigma-2 \mu}\left\{I(0, \bar{x})+I\left(\bar{x}, x_{c}\right)+I\left(x_{c}, x\right)\right\}
$$


is readily obtained. The application of Lemmas 1 and 2 to the respective terms on the right is direct. If it is tentatively assumed that Lemma 3 is applicable in similar fashion to the final term it may be concluded that

$$
\left|\xi^{1 / 2-\mu} Y_{j}^{(n)}(x)\right| \leqq M^{n}\left\{M_{1}|\rho|^{\sigma-4 \mu}+M_{2}|\rho|^{\sigma-\sigma_{1}}+M_{3}|\rho|^{\sigma-1}\right\},
$$

and hence that the relation (37) is valid provided, as in Case 2, the chosen value of $\sigma$ does not exceed $\sigma_{1}$.

The application of the Lemma 3 in the manner provisionally assumed above is dependent, of course, upon the fulfillment of the hypotheses upon which the lemma is based, namely, for the case in hand, upon the convergence of the integral of the function $\Psi^{2}(t)|\theta(t)| /\{\Phi(t)\}^{1-2 \mu}$. This function reduces in virtue of the formulas (5) to the form $|\theta(t)| / \phi(t)$, and it is clear, therefore, that the conclusions reached above are substantiable if, when the interval $I$ is infinite, the list of hypotheses of $\$ 2$ is augmented by the following addition.

(v) The coefficients of the given differential equation are such that the integral

$$
\int \frac{\theta(t)}{\phi(t)} d t
$$

converges absolutely when extended over those portions of the interval I on which $|t|$ exceeds some positive constant.

In summary, then, the relations (37) have been established firstly for $|\xi| \leqq N$ if $\sigma=4 \mu$, and secondly for $|\xi|>N$ if $\sigma=\sigma_{1}$. It follows directly, of course, that the infinite series in the relation (27) converges uniformly as to $x$ when $|\rho|$ is sufficiently large, and that the relation in question accordingly represents a solution of the equation (24b). Specifically, therefore, there exists a solution of this equation which satisfies the relations

$$
\begin{aligned}
U_{j}(x) & =Y_{j}(x)+\sum_{n=1}^{\infty} \frac{E_{n}(x, \rho)}{\rho^{4 n \mu}}, \text { when }|\xi| \leqq N, \\
\xi^{1 / 2-\mu} U_{j}(x) & =\xi^{1 / 2-\mu} Y_{j}(x)+\sum_{n=1}^{\infty} \frac{E_{n}(x, \rho)}{\rho^{n \sigma_{1}}}, \text { when }|\xi|>N,
\end{aligned}
$$

the symbols $E$ being of the significance explained as follows.

The terms of the infinite series in the relation (27) are successively expressed by the formula (28) in terms of functions either initially known or previously determined. They are, therefore, known in the sense that they are expressible by specifiable formulas, and as such are, at least theoretically, computable. These observed facts together with the boundedness of the functions in question constitute the essential features with respect to which the series in the relation (27) and those series later to be derived from (27) 
are of interest from the viewpoint of the discussion of the present paper. An economy of thought and formulas may, therefore, be achieved by the use of the letter $E$ as a generic symbol in the following sense. The symbol $E$ shall designate merely some computable function which is bounded for the ranges of its arguments under consideration. In a given formula different functions $E$ will be distinguished by the use of subscripts. There is to be no presumption, however, that the same symbol in different formulas designates the same function. As a case in point it is evident that the functions similarly designated in the two equations (40) are not the same.

By means of the substitutions (25) the relations (40) are now readily made to yield the formulas for the solution $u_{k, j}(x)$ of the given differential equation. It may be observed to begin with that when $|\xi| \leqq N$ the functions $\Psi(x)$ and $e^{ \pm i \xi}$ are bounded. Hence the first of the relations (40) reduces to the formula

$$
u_{k, j}(x)=y_{k, j}(x)+\sum_{n=1}^{\infty} \frac{E_{n}(x, \rho)}{\rho^{4 n \mu}}, \text { for }|\xi| \leqq N, \text { and } \xi \text { in } \Xi_{k, l}
$$

The second of the relations (40) yields in the same way the formula

$$
\begin{gathered}
u_{k, j}(x)=y_{k, j}(x)+\Psi(x) \xi^{\mu-1 / 2} e^{ \pm i \xi} \sum_{n=1}^{\infty} \frac{E_{n}(x, \rho)}{\rho^{n \sigma_{1}}} \\
\quad \text { for }|\xi|>N, \text { and } \xi \text { in } \Xi_{k, l}
\end{gathered}
$$

and if the asymptotic expression $\{20)$ is substituted for $y_{k, j}(x)$ on the right of this equation there results the asymptotic relation

(41c) $u_{k, j}(x) \sim \Psi(x) \xi^{\mu-1 / 2} e^{ \pm i \xi}\left\{1+\sum_{n=1}^{\infty} \frac{E_{n}(x, \rho)}{\rho^{n \sigma_{1}}}+\frac{c_{n}^{k, j}}{\xi^{n}}\right\}$, for $\xi$ in $\Xi_{k, l}$.

The formulas (41a), (41b), and (41c) have been established, it will be recalled, under the supposition that $j=j^{\prime}$. They embody the results for which the deductions of the present section were primarily made.

It was observed in $\$ 5$ that the formulas there derived remain valid if the quantities $u_{k, j}$ and $y_{k, j}$, where they occur with the argument $x$, are replaced throughout by $\bar{u}_{k, j}(x)$ and $\bar{y}_{k, j}(x)$ respectively. The persisting validity of the relation (34) under this change is contingent upon the simultaneous substitution of the factor $\xi^{(\mu-1 / 2) s}$ in place of $\xi^{(1 / 2-\mu) s}$. Inasmuch as all conclusions of the present section are based on the relation (34) and the formulas of $\$ 5$, it is therefore clear that a repetition of the arguments made with the substitutions of quantities noted will lead to formulas for the quantity $\bar{u}_{k, j}(x)$ analogous to those for $u_{k, j}(x)$ explicitly derived above. It will 
suffice, therefore, merely to note the formulas which are obtained in the manner indicated, namely

$$
\begin{aligned}
& \text { (42a) } \bar{u}_{k, j}(x)=\bar{y}_{k, j}(x)+\sum_{n=1}^{\infty} \frac{E_{n}(x, \rho)}{\rho^{4 n \mu}}, \text { for }|\xi| \leqq N \text {, and } \xi \text { in } \Xi_{k, l}, \\
& \text { (42b) } \bar{u}_{k, j}(x)=\bar{y}_{k, j}(x)+\Psi(x) \xi^{1 / 2-\mu} e^{ \pm i \xi} \sum_{n=1}^{\infty} \frac{E_{n}(x, \rho)}{\rho^{n \sigma_{1}}}, \text { for }|\xi|>N \text {, and } \xi \text { in } \Xi_{k, l}, \\
& \text { (42c) } \pm \bar{u}_{k, j}(x) \sim \Psi(x) \xi^{1 / 2-\mu} e^{ \pm i \xi}\left\{1+\sum_{n=1}^{\infty} \frac{E_{n}(x, \rho)}{\rho^{n \sigma_{1}}}+\frac{\bar{c}_{n}^{k, j}}{\xi^{n}}\right\} \text { for } \xi \text { in } \Xi_{k, l} .
\end{aligned}
$$

Theorem 3. If $x$ and $\xi$ are confined respectively to an interval $I^{*}$ and $a$ quadrant $\Xi_{k, l}$, and if for these values $y_{k, j^{\prime}}(x)$ is the dominant solution of the related equation, then there exists a solution $u_{k, i^{\prime}}(x)$ of the given differential equation which is described for the values in question by the various formulas (41) and (42). If the interval $I^{*}$ is infinite, the hypothesis (v) must be fulfilled.

8. The sub-dominant solution for $\xi$ in a quadrant $\Xi_{k, l}$. Theorem 3 asserts in brief the existence of a solution of the given differential equation which is asymptotically represented by the dominant solution of the related equation. It is to be shown in this section that the sub-dominant solution of the related equation likewise serves as the asymptotic representative of certain solutions of the equation given. As in the case of the preceding section, the considerations are to be based upon the relations formally obtained in $\$ 5$. These relations will be considered now, however, for the subscript value to be designated by $j=j^{\prime \prime}$, and associated with the sub-dominant one of the solutions $y_{k, j}(x)$.

The value of the constant $x_{0}$, unspecified in the formulas of $\$ 5$, was chosen for the case $j=j^{\prime}$ in $\$ 7$. For the case $j=j^{\prime \prime}$ now in hand no single choice of this constant is of exclusive advantage, and it is primarily due to this fact that a difference will be observable in the results of this section and those of the preceding one. The various choices of $x_{0}$ are governed, however, by certain general considerations which will now be made.

The inequalities (34) are valid for either value of $j$, and hence in particular for $j=j^{\prime \prime}$. The boundedness of the quantity within the brace of formula (26) depends, therefore, upon the function $\exp \{\mp 2 i(\xi-\tau)\}$, the sign involved to be chosen as that associated with the value $j=j^{\prime \prime}$. Now for this value of $j$ the corresponding exponent in the formula (20) has a negative real part. If $x$ and $t$ are both taken to lie on the interval $I^{*}$, it follows that the real part of the exponent in formula (26) will also be negative provided

$$
\arg [i(\xi-\tau)]=\arg [-i \xi]
$$


a condition which is fulfilled if and only if $|\tau| \geqq|\xi|$, i.e., if $|t| \geqq|x|$. Inasmuch as the variable of integration $t$ in formulas (24b) and (28) ranges over the interval bounded by $x$ and $x_{0}$, it is clear that when $x_{0}$ is chosen the considerations must be confined to those values of $x$ on the interval $I^{*}$ for which $0 \leqq|x| \leqq\left|x_{0}\right|$. With this restriction the inequalities (36) are readily found to be valid for $j=j^{\prime \prime}$ provided $|t| \geqq|x|$.

With any choice of $x_{0}$ on the interval $I^{*}$, and $x$ restricted as determined above, it follows from the assumption (37a), as in the preceding section, that the function (38) satisfies the hypotheses imposed in $\$ 6$ upon the function $H(x, t, \rho)$. Hence the expression of the formula (28) by means of integrals with the structure of those considered in the lemmas of $\$ 6$ is possible, and with appropriate choices of the value $\sigma$ the relations (37) may be established as follows.

Case 1. $x_{0}=\bar{x}$. With this choice of $x_{0},|\xi| \leqq N$. The function (38) with $s=0$ may be employed, therefore, in the part of $H(x, t, \rho)$ and as a result the relation (28) may be written in the form

$$
Y_{j}^{(n)}(x)=M^{n} \rho^{\sigma-2 \mu} I(x, \bar{x}) .
$$

By Lemma 1 this leads to the inequalities (37) provided $\sigma$ is chosen not greater than $4 \mu$. It may be concluded, then, that the infinite series in the relation (27) represents a solution of the equation (24b), and that it may be written in the form of the first of the relations (40). It is thus found that there exists a solution of the given differential equation which is described by the formulas (41a) and (42a) for the value $j=j^{\prime \prime}$.

TheOREM $4 \mathrm{a}$. If $x$ and $\xi$ are confined respectively to the interval $I^{*}$ and the quadrant $\Xi_{k, l}$ and if for these values $y_{k, j^{\prime \prime}}(x)$ is the sub-dominant solution of the related equation, then there exists a solution $u_{k, j^{\prime \prime}}(x)$ of the given differential equation which is described by the formulas (41a) and (42a).

It should be remarked that Theorem 4 a makes no assertion as to the form of the solution concerned for values of $x$ such that $|\xi|>N$.

Case 2. $x_{0}=x_{c}$, where $x_{c}$ is any fixed point of the interval $I^{*}$. The procedure is now familiar. The formula (28) may be written for $x$ on the respective ranges shown in the forms

$$
\begin{aligned}
\xi^{1 / 2-\mu} Y_{j}^{(n)}(x) & =M^{n} \rho^{\sigma-2 \mu} I\left(x, x_{c}\right), \text { when }|\bar{x}|<|x| \leqq\left|x_{c}\right| \\
Y_{j}^{(n)}(x) & =M^{n} \rho^{\sigma-2 \mu}\left\{I(x, \bar{x})+I\left(\bar{x}, x_{c}\right)\right\}, \text { when } 0 \leqq|x| \leqq|\bar{x}| .
\end{aligned}
$$

The conclusion (37) follows from Lemmas 1 and 2 provided $\sigma \leqq \sigma_{1}$. If the interval $I^{*}$ is finite all values of $x$ may be included in Case 2 by choosing $x_{e}$ as the end point of $I^{*}$. 
It is to be especially noted that by formula (28) the quantities $Y_{j^{\prime \prime}}{ }^{(n)}(x)$ are essentially dependent upon the value $x_{0}$, and that these quantities in Case 2 are, therefore, not identical with those in Case 1. Despite this fact it will not be found necessary to resort to a distinguishing notation for the solutions concerned in the respective cases.

If the interval $I^{*}$ is infinite, it will be supposed as in $\$ 7$ that the hypothesis (v) is fulfilled. The considerations may be extended, then, also to the following case.

Case 3. $\left|x_{0}\right|=\infty$. In this case the formula (28) may be written for $x$ on the respective ranges shown in the forms

$$
\begin{aligned}
& \xi^{1 / 2-\mu} Y_{j}^{(n)}(x)=M^{n} \rho^{\sigma-2 \mu} I(x, \infty) \text {, when }\left|x_{c}\right|<|x|<\infty, \\
& \xi^{1 / 2-\mu} Y_{j}^{(n)}(x)=M^{n} \rho^{\sigma-2 \mu}\left\{I\left(x, x_{c}\right)+I\left(x_{c}, \infty\right)\right\} \text {, when }|\bar{x}|<|x| \leqq\left|x_{c}\right|, \\
& \quad Y_{j}^{(n)}(x)=M^{n} \rho^{\sigma-2 \mu}\left\{I(x, \bar{x})+I\left(\bar{x}, x_{c}\right)+I\left(x_{c}, \infty\right)\right\} \text {, when } 0<|x| \leqq|\bar{x}| .
\end{aligned}
$$

An application of the lemmas establishes the relations (37) under the assumption that $\sigma \leqq \sigma_{1}$.

The uniform convergence of the infinite series in the relation (27) follows in both Cases 2 and 3 when $|\rho|$ is sufficiently large, and the solution $U_{j}(x)$ is found to satisfy the second of the relations (40). The first of the relations (40) must, however, be replaced in these cases by the equation

$$
U_{j}(x)=Y_{j}(x)+\sum_{n=1}^{\infty} \frac{E_{n}(x, \rho)}{\rho^{n \sigma_{1}}}, \text { for }|\xi| \leqq N \text {, and } \xi \text { in } \Xi_{k, l} .
$$

Hence it may be concluded that the corresponding solution of the equation (3a) satisfies the relations (41b), (41c) and (42b), (42c), whereas the relations (41a) and (42a) must be replaced by the equations

$$
\begin{aligned}
& u_{k, j}(x)=y_{k, j}(x)+\sum_{n=1}^{\infty} \frac{E_{n}(x, \rho)}{\rho^{n \sigma_{1}}}, \text { for }|\xi| \leqq N, \text { and } \xi \text { in } \Xi_{k, l}, \\
& \bar{u}_{k, j}(x)=\bar{y}_{k, j}(x)+\sum_{n=1}^{\infty} \frac{E_{n}(x, \rho)}{\rho^{n \sigma_{1}}}, \text { for }|\xi| \leqq N, \text { and } \xi \text { in } \Xi_{k, l} .
\end{aligned}
$$

THEOREM 4b. If $x$ and $\xi$ are respectively restricted to the interval $I^{*}$ and the quadrant $\Xi_{k, l}$, and if for these values $y_{k, j^{\prime \prime}}(x)$ is the sub-dominant solution of the related equation, then there exists a solution $u_{k, j^{\prime \prime}}(x)$ of the given equation which is described by the formulas (41b), (41c), (41d) and (42b), (42c), (42d).

9. The general solution of the given equation. The discussion has been concerned hitherto with the form of certain particular solutions of the given and related equations for values of $x$ and $\xi$ peculiarly restricted. It is pro- 
posed now to extend the considerations to the general solutions of these equations and to general values of the parameter and variable.

In the foregoing sections it was convenient to base the deductions upon the particular sets of solutions $y_{k, j}(x)$, because of the especial simplicity of the corresponding asymptotic forms for the values of $\xi$ on the restricted ranges considered. The advantage in this choice naturally fails to persist when general values of $\xi$ are drawn into account. It is for this reason convenient to introduce at this point as a basis for the continuing discussion a set of solutions distinct from any of those hitherto used. These solutions $y_{j}(x)$ and their associated quantities $\bar{y}_{j}(x)$ will be defined by the formulas

$$
\begin{aligned}
& y_{j}(x)=\Psi(x) \xi^{\mu} J_{\mp \mu}(\xi), \\
& \bar{y}_{j}(x)= \pm i \Psi(x) \xi^{1-\mu} J_{ \pm(1-\mu)}(\xi), j=1,2,
\end{aligned}
$$

in which the symbols $J$ designate the familiar Bessel functions of the first kind. The suitability of this definition is warranted by Theorem 1 . It is, moreover, directly verified that the quantities $\bar{y}_{j}(x)$ as given in (43) are related to the respective solutions $y_{j}(x)$, by the formula obtainable from (19) by deletion of the subscripts $k$. The significance and purpose of the quantities $\bar{y}_{j}(x)$ and their relation to the derivatives $y_{j}^{\prime}(x)$, is familiar from the rôle of the quantities (19) in the earlier discussion.

The point $x=0$ is an ordinary point for both the given and related equations. Hence the values $y_{j}(0), \bar{y}_{j}(0), j=1,2$, are completely determinate, and a pair of solutions $u_{1}(x), u_{2}(x)$ of the given differential equation is uniquely defined by the conditions

$$
u_{j}(0)=y_{j}(0), \bar{u}_{j}(0)=\bar{y}_{j}(0), j=1,2,
$$

the quantities $\bar{u}_{j}(x)$ being obtainable from the formula (29) by suppression of the subscripts $k$. In virtue of the familiar relation [W, p. 40]

$$
\lim _{z \rightarrow 0} z^{\eta} J_{-\eta}(z)=\frac{2^{\eta}}{\Gamma(1-\eta)},
$$

the specific values which thus fix the solutions $u_{j}(x)$ are readily found to be

$$
\begin{array}{ll}
u_{1}(0)=\frac{2^{\mu} \Psi(0)}{\Gamma(1-\mu)}, & \bar{u}_{1}(0)=0 \\
u_{2}(0)=0, & \bar{u}_{2}(0)=\frac{2^{1-\mu} \Psi(0)}{i \Gamma(\mu)} .
\end{array}
$$

It is evident that the solutions $u_{1}(x)$ and $u_{2}(x)$ are linearly independent. Hence the general solution, $u(x)$, of the equation given can be expressed 
linearly in terms of them. The explicit formula is in fact easily obtained, and is the following: if the solution $u(x)$ takes at $x=0$ the values

$$
u(0)=C, \bar{u}(0)=\bar{C},
$$

then

$$
u(x) \equiv \frac{\Gamma(1-\mu) C}{2^{\mu} \Psi(0)} u_{1}(x)+\frac{i \Gamma(\mu) \bar{C}}{2^{1-\mu} \Psi(0)} u_{2}(x) .
$$

With the formula (47b) at hand it is clear that a determination of form for the solutions $u_{1}(x)$ and $u_{2}(x)$ suffices in every respect for a corresponding determination of form for the general solution of the equation given.

Let any value $x$ on the interval $I$ be chosen, and let $\rho$ be located at pleasure in the complex plane subject to the condition that $|\rho|$ be sufficiently large. The corresponding value of $\xi$ lies in some one of the quadrants (14), and the designation of this quadrant by $\Xi_{k, l}$ has merely the significance of an assignment of values to $k$ and $l$. For the value of $k$ thus fixed upon and for the value of $\xi$ in question the solutions $u_{k, j}(x)$ given by the Theorems 3 and either $4 \mathrm{a}$, or $4 \mathrm{~b}$, have the forms respectively deduced for them in $\$ \S 7$, and 8 , and are given accordingly by the appropriate formulas (41) and (42). Between any pair of these solutions and the solutions $u_{j}(x)$ considered above there exists, of course, a set of identical relations

$$
\begin{aligned}
& u_{l}(x)=a_{l, 1}^{(k)} u_{k, 1}(x)+a_{l, 2}^{(k)} u_{k, 2}(x), \\
& \bar{u}_{l}(x)=a_{l, 1}^{(k)} \bar{u}_{k, 1}(x)+a_{l, 2}^{(k)} \bar{u}_{k, 2}(x),
\end{aligned}
$$$$
l=1,2,
$$

with coefficients $a_{l, j}{ }^{(k)}$ which are free from $x$ but which naturally depend upon the solutions $u_{k, j}(x)$ involved. The existence of an analogous set of relations involving the corresponding solutions of the related equation, i.e.,

$$
\begin{aligned}
& y_{l}(x)=c_{l, 1}^{(k)} y_{k, 1}(x)+c_{l, 2}^{(k)} y_{k, 2}(x), \\
& \bar{y}_{l}(x)=c_{l, 1}^{(k)} \bar{y}_{k, 1}(x)+c_{l, 2}^{(k)} \bar{y}_{k, 2}(x),
\end{aligned} \quad l=1,2,
$$

is evident.

The coefficients in the identities (48a) and (48b) are easily calculated by setting $x=0$ and solving the resulting system of algebraic equations. In virtue of the relations (44) the resulting values may be written

$$
\begin{aligned}
a_{l, 3-j}^{(k)} & =\frac{\mp\left\{y_{l}(0) \bar{u}_{k, j}(0)-\bar{y}_{l}(0) u_{k, j}(0)\right\}}{D\left(u_{k, 1}(0), u_{k, 2}(0)\right)}, \\
c_{l, 3-j}^{(k)} & =\frac{\mp\left\{y_{l}(0) \bar{y}_{k, j}(0)-\bar{y}_{l}(0) y_{k, j}(0)\right\}}{D\left(y_{k, 1}(0), y_{k, 2}(0)\right)},
\end{aligned}
$$


the symbol $D$ having the significance given it in formula (22a). The evident similarity of structure of the right hand members of formulas (49a) and (49b) will be used as a basis for deducing also a similarity of values of the coefficients which they represent.

Let the attention be given first to a consideration of those values of $x$ and $\rho$ for which $|\xi| \leqq N$. The solutions $u_{k, j}(x)$ in formulas (48a) and (49a) are in this case conveniently taken as those described by the Theorems 3 and 4a. The formulas (41a) and (42a) may accordingly be drawn upon for an evaluation of the quantities $u_{k, j}(0)$ and $\bar{u}_{k, j}(0)$, and if these values are substituted into the relations (49a) and the resulting formulas are compared with the relations $(49 \mathrm{~b})$, it is found that

$$
a_{l, j}^{(k)}=c_{l, j}^{(k)}+\sum_{n=1}^{\infty} \frac{E_{n}(\rho)}{\rho^{4 n \mu}}, \quad j, l=1,2 .
$$

With these coefficient values, and with the values of $u_{k, j}(x)$ and $\bar{u}_{k, j}(x)$ given by the formulas (41a) and (42a), the identities (48a) are found upon comparison with (48b) to reduce to the form

$$
\begin{array}{ll}
u_{l}(x)=y_{l}(x)+\sum_{n=1}^{\infty} \frac{E_{n}(x, \rho)}{\rho^{4 n \mu}}, & \\
\bar{u}_{l}(x)=\bar{y}_{l}(x)+\sum_{n=1}^{\infty} \frac{E_{n}(x, \rho)}{\rho^{4 n \mu}}, & l=1,2 .
\end{array}
$$

Inasmuch as the quantities $y$ in these equations are those of the formulas (43), the results obtained may be summarized as follows:

TheOREM 5. The solutions $u_{1}(x), u_{2}(x)$, of the given differential equation, which at $x=0$ take on the values (46), are described by the formulas

$$
\begin{aligned}
& u_{j}(x)=\Psi(x) \xi^{\mu} J_{\mp \mu}(\xi)+\sum_{n=1}^{\infty} \frac{E_{n}(x, \rho)}{\rho^{4 n \mu}} \\
& \bar{u}_{j}(x)= \pm i \Psi(x) \xi^{1-\mu} J_{ \pm(1-\mu)}(\xi)+\sum_{n=1}^{\infty} \frac{E_{n}(x, \rho)}{\rho^{4 n \mu}}, \quad j=1,2,
\end{aligned}
$$

for all values of $x$ and $\rho$ subject to the relation $|\xi| \leqq N$.

Let the attention be turned now to a consideration of the values of $x$ and $\rho$ for which $|\xi|>N$. The procedure to be followed is in its initial stage similar to that followed above, with the difference, however, that the solutions $u_{k, j}(x)$. involved in the formulas (48a) and (49a) are to be looked upon as those given by the Theorems 3 and $4 \mathrm{~b}$ rather than by Theorems 3 and $4 \mathrm{a}$ as heretofore. This change in the point of view is seen directly to be permissible, 
for the solutions on the left of the identities (48a) are uniquely determined by the values (46) and are, therefore, in particular independent of the solutions used on the right. A change in this set of solutions requires, of course, a compensating change in the corresponding coefficients. This, however, is entirely accounted for if the solutions used in formulas (48a) and (49a) are the same.

The solutions $u_{k, j}(x)$ described by Theorems 3 and $4 \mathrm{~b}$ are given when $|\xi| \leqq N$, and hence, in particular when $x=0$, by the relations (41d). A substitution of the values thus obtained into the formulas (49a) and a comparison of the resulting relations with the formulas (49b) show in a manner already familiar that in this case

$$
a_{l, j}^{(k)}=c_{l, j}^{(k)}+\sum_{n=1}^{\infty} \frac{E_{n}(\rho)}{\rho^{n \sigma_{1}}}, \quad j, l=1,2 .
$$

The explicit values of the coefficients $c$ on the right of the equations (51) are deducible without difficulty from a comparison of the identities (48b) with certain standard and well known formulas from the theory of the Bessel functions. Thus if the forms (43) and (20) are substituted respectively on the left and right of the relations (48b) the formulas which result may be written

$$
\begin{array}{r}
J_{\mp \mu}(\xi) \sim \frac{1}{\xi^{1 / 2}}\left\{c_{j, 1}^{(k)} e^{i \xi}\left[1+\sum_{n=1}^{\infty} \frac{c_{n}^{k, 1}}{\xi^{n}}\right]+c_{j, 2}^{(k)} e^{-i \xi}\left[1+\sum_{n=1}^{\infty} \frac{c_{n}^{k, 2}}{\xi^{n}}\right]\right\}, \\
j=1,2 .
\end{array}
$$

Inasmuch as the asymptotic relations here represented are explicitly familiar [W, p. 202] an identification of the coefficients $c$ is possible. Since $\xi$ lies in the half-plane $\Xi_{k}$, it is found in this way that

$$
\frac{1}{(2 \pi)^{1 / 2}} e^{(k+1 / 2)(1 / 2 \mp \mu) \pi i}=\left\{\begin{array}{l}
c_{j, 1}^{(k)}=c_{j, 2}^{(k)} e^{(1 / 2 \mp \mu) \pi i}, \text { when } k \text { is odd } \\
c_{j, 2}^{(k)}=c_{j, 1}^{(k)} e^{(1 / 2 \mp \mu) \pi i}, \text { when } k \text { is even }
\end{array}\right.
$$

These are the values of the coefficients which occur on the right of the equations (51).

The deduction of the asymptotic forms of the solutions $u_{1}(x)$ and $u_{2}(x)$ for the arbitrarily chosen configuration of values $x$ and $\rho$ is now at hand. The quantities on the right of the identities (48a) are given when $|\xi|>N$ by the forms (41c) and (42c), and these forms may now be substituted. Since the corresponding coefficients are evaluated by the set of relations (51) and (52b) it is thus found finally that the solutions in question are given for all values of $x$ and $\rho$ subject to $|\xi|>N$ by the asymptotic formulas 


$$
\begin{array}{r}
u_{j}(x) \sim \Psi(x) \xi^{\mu-1 / 2}\left\{a_{j, 1} e^{i \xi}\left[1+\sum_{n=1}^{\infty} \frac{E_{n 1}(x, \rho)}{\rho^{n \sigma_{1}}}+\frac{E_{n 2}}{\xi^{n}}\right]\right. \\
\left.+a_{j, 2} e^{-i \xi}\left[1+\sum_{n=1}^{\infty} \frac{E_{n 3}(x, \rho)}{\rho^{n \sigma_{1}}}+\frac{E_{n 4}}{\xi^{n}}\right]\right\}, \\
\bar{u}_{j}(x) \sim \Psi(x) \xi^{1 / 2-\mu}\left\{a_{j, 1} e^{i \xi}\left[1+\sum_{n=1}^{\infty} \frac{\bar{E}_{n 1}(x, \rho)}{\rho^{n \sigma_{1}}}+\frac{\bar{E}_{n 2}}{\xi^{n}}\right]\right. \\
\left.-a_{j, 2} e^{-i \xi}\left[1+\sum_{n=1}^{\infty} \frac{\bar{E}_{n 3}(x, \rho)}{\rho^{n \sigma_{1}}}+\frac{\bar{E}_{n 4}}{\xi^{n}}\right]\right\},
\end{array}
$$

with $a_{j, i}=a_{j, i}{ }^{(k)}$ when $\xi$ lies in the half-plane $\Xi_{k}$, and

$$
\begin{aligned}
a_{j, 1}^{(2 p)} & =\frac{1}{(2 \pi)^{1 / 2}} e^{(2 p-1 / 2)(1 / 2 \mp \mu) \pi i}+\sum_{n=1}^{\infty} \frac{E_{n}(\rho)}{\rho^{n \sigma_{1}}}, \\
a_{j, 2}^{(2 p)} & =\frac{1}{(2 \pi)^{1 / 2}} e^{(2 p+1 / 2)(1 / 2 \mp \mu) \pi i}+\sum_{n=1}^{\infty} \frac{E_{n}(\rho)}{\rho^{n \sigma_{1}}}, \\
a_{j, 1}^{(2 p+1)} & =\frac{1}{(2 \pi)^{1 / 2}} e^{(2 p+3 / 2)(1 / 2 \mp \mu) \pi i}+\sum_{n=1}^{\infty} \frac{E_{n}(\rho)}{\rho^{n \sigma_{1}}}, \\
a_{j, 2}^{(2 p+1)} & =\frac{1}{(2 \pi)^{1 / 2}} e^{(2 p+1 / 2)(1 / 2 \mp \mu) \pi i}+\sum_{n=1}^{\infty} \frac{E_{n}(\rho)}{\rho^{n \sigma_{1}}}, \quad j=1,2 .
\end{aligned}
$$

The results thus obtained may be summarized in the following and final theorem.

THEOREM 6. The solutions $u_{1}(x), u_{2}(x)$ of the given differential equation, which at $x=0$ take the values (46), are asymptotically described by the formulas (53a), (53b), for all values of $x$ and $\rho$ subject to the relation $|\xi|>N$.

Theorems 5 and 6 are to be considered as culminating the general theoretical deductions to which this paper has been given. A brief critique only of the concluding formulas (53) is still in order. The asymptotic formulas for the Bessel functions given above in (52a) are familiarly known to be subject to abrupt changes of the coefficients when the complex argument $\xi$ passes from any one to any other half-plane $\Xi_{k}$. This characteristic, discovered by Stokes in 1857 [W, p. 201] and designated as the Stokes' phenomenon, is qualitatively displayed and quantitatively determined by the dependence of the formulas (52b) upon the value of $k$. It is now evident from the formulas (53a) and (53b) that the asymptotic forms of the solutions $u_{i}(x)$ of any given differential equation (3) are likewise and in similar manner dependent upon the location of $\xi$ in the complex plane, namely, upon the configuration of the values of $x$ and $\rho$. The Stokes' phenomenon, far from 
being peculiar to the Bessel functions, is thus revealed as the special manifestation of a characteristic common to the solutions of all differential equations of the types (1) or (3).

Aside from the dependence of the coefficients (53b) upon the value of $p$ as noted, the further dependence of these coefficients upon the value of $\mu$ should be observed. The value $\mu$, it will be recalled, is determined by the degree to which $\phi^{2}(x)$, the coefficient of the parameter in the given differential equation, vanishes at its zero on the interval $I$. The character of this zero is, therefore, seen to be determinative of the law by which the change in asymptotic forms specific of the Stokes' phenomenon takes place with changing values of $x$ and $\rho$.

The border line case $\mu=\frac{1}{2}$ is of sufficient peculiarity to deserve a word of mention. This value of $\mu$ is associated with the case in which the coefficient $\phi^{2}(x)$ vanishes to the degree zero, namely, does not vanish at all, on the interval under consideration. It is easily verified that when $\mu=\frac{1}{2}$ the dependence of the coefficients (53b) upon the value of $p$ is only apparent, and hence in this case, and in this case only, does the Stokes' phenomenon fail to arise.*

\section{PART II}

AN APPLICATION TO THE THEORY OF THE BESSEL FUNCTIONS OF LARGE ORDER

10. Introduction. The theory constructed for the general differential equation of type (3) in the preceding sections leans heavily for its results upon the classical theory of the Bessel functions of the small real orders $\mu$ and $1-\mu$. It is almost in the manner of compensation, therefore, that it finds an extensive application in the theory of the Bessel functions of orders real and large. It is to this application that the discussion of the present part of the paper will be devoted.

The theory of the Bessel functions, it will be recalled, customarily deals with the derivation of asymptotic formulas in two distinct ramifications. To quote on this point from Watson's treatise [W, p. 194], "There are really two aspects of the problem to be considered; the investigation when [the order] is large is very different from the investigation when [the order] is not large. The former investigation is, in every respect, of a more recondite character than the latter..."

Despite this partition of the theory and the associated differentiation in methods of investigation, it is nevertheless found that certain of the resulting formulas relate intimately the functions of the two categories separately

* This is, of course, in harmony with the elementary fact that if $\phi^{2}$ is a constant and the equation is $u^{\prime \prime}+\rho^{2} \phi^{2} u=0$, then the expressions $u_{j}=\exp ( \pm i \rho \phi x)$ are explicit solutions, and are in consequence of the same iorm for all values of $x$ and $\rho$. 
considered. This fact is accounted for in an entirely natural manner in virtue of the application of the theory of the present paper to the problem at hand. The derivation of the results through the medium of this application should, moreover, hardly be characterized as "recondite." The theory itself, as will have been found, is essentially elementary in character, and the application is correspondingly simple.

Consistent with the policy pursued in the deductions hitherto, no effort has in general been spent toward carrying the explicit computation of the formulas beyond that of the leading terms. The principal point of interest would seem to lie in the fact that the various formulas, which frequently depend upon widely diverse methods for their derivation, spring here, as from a single and unified source, from the formulas of $\$ 9$. Certain differences in the formulas here obtained from those usually given for the functions in question will be noted as they arise.

11. The transformed Bessel's equation. The differential equation

$$
u^{\prime \prime}(x)+\rho^{2}\left\{e^{2 x}-1\right\} u(x)=0
$$

is transformed by the substitution $z=\rho e^{x}$ into the Bessel's equation with parameter $\rho$ and argument $z$. Its solutions, therefore, include in particular the functions

$$
J_{\rho}\left(\rho e^{x}\right), Y_{\rho}\left(\rho e^{x}\right), H_{\rho}^{(1)}\left(\rho e^{x}\right),
$$

respectively Bessel functions of the first, second, and third kinds. Any two of the functions (55) are linearly independent, and hence any Bessel function of like order and argument can be expressed in terms of them. Between the three functions (55) there accordingly exists an identical relation, and this is known to be

$$
J_{\rho}(z)+i Y_{\rho}(z)-H_{\rho}^{(1)}(z)=0 .
$$

The equation (54) is obviously of the type (3). Inasmuch as the coefficient $\phi^{2}(x)$ is in this case the function $e^{2 x}-1$, which vanishes to the degree 1 at $x=0$, the following formulas are readily found for the explicit identification of the respective quantities, all of which are of familiar import in the general theory:

$$
\begin{aligned}
\mu & =\frac{1}{3}, \sigma_{1}=1, \\
\phi(x) & =\left(e^{2 x}-1\right)^{1 / 2}, \\
\Phi(x) & =\phi(x)-\tan ^{-1} \phi(x), \\
\Psi(x) & =\frac{\{\Phi(x)\}^{1 / 6}}{\{\phi(x)\}^{1 / 2}} \\
\chi(x) & \equiv 0 .
\end{aligned}
$$


The hypotheses (i) to (iv) of $\S 2$ are found at a glance to be satisfied with the interval $I$ chosen as the entire axis of $x$, i.e., $-\infty<x<\infty$. The admissibility of this choice of an infinite interval and the corresponding availability of the general results for all values of $x$ depend further, therefore, only upon the applicability of the hypothesis (v) of $\$ 7$ to the case in hand. This applicability may be investigated without difficulty by the procedure outlined as follows. For values of $x$ which are numerically large and respectively positive or negative the function $\Omega(x)$ defined in formula (5) may be expanded in negative or positive powers of $e^{x}$. It is found in this way that for $x>0$,

$$
\Omega(x)=1-\frac{\tan ^{-1} \phi(x)}{\phi(x)}=1-\frac{\pi}{2} e^{-x}+e^{-2 x}+\cdots,
$$

while for $x<0$,

$$
\begin{aligned}
\Omega(x) & =1+\frac{x-\log [1+i \phi(x)]}{i \phi(x)} \\
& =x\left\{1+\frac{1}{2} e^{2 x}+\cdots\right\}+(1-\log 2)\left\{1+\frac{1}{2} e^{2 x}+\cdots\right\} .
\end{aligned}
$$

By means of the formulas (12) and (23) it is now readily computed that for positive values of $x, \theta(x)=O(1)$, and in consequence $\theta(x) / \phi(x)=O\left(e^{-x}\right)$, whereas when $x$ is negative $\theta(x)=O\left(x^{-2}\right)$, and $\theta(x) / \phi(x)=O\left(x^{-2}\right)$. The hypothesis ( $v$ ) accordingly demands the convergence of a pair of integrals of the type

$$
\int_{c}^{\infty} O\left(e^{-x}\right) d x, \int_{-\infty}^{-c} O\left(\frac{1}{x^{2}}\right) d x \text {, with } c>0,
$$

and this requirement is clearly fulfilled.

It will be understood throughout the following discussion that the parameter $\rho$ is taken to be large, real, and positive. The value of $e^{x}$ is greater than or less than unity according as $x$ is positive or negative. It is convenient to introduce in place of $x$ on these two ranges the familiar real variables $\beta$ and $\alpha$ defined by the relations

$$
e^{x}=\sec \beta, \text { for } x \geqq 0 ; e^{x}=\operatorname{sech} \alpha, \text { for } x<0 .
$$

In terms of these variables it is readily found that

$$
\begin{aligned}
\phi(x) & =\tan \beta \\
\xi & =\rho[\tan \beta-\beta], \\
\Psi(x) & =\frac{[\tan \beta-\beta]^{1 / 6}}{[\tan \beta]^{1 / 2}}, \text { for } x \geqq 0,
\end{aligned}
$$


and

$$
\begin{aligned}
\phi(x) & =i \tanh \alpha \\
\xi & =i^{3} \rho[\alpha-\tanh \alpha] \\
\Psi(x) & =\frac{[\alpha-\tanh \alpha]^{1 / 6}}{[\tanh \alpha]^{1 / 2}}, \text { for } x<0 .
\end{aligned}
$$

The formulas of $\$ 9$ would readily yield the description of the general solution of the equation (54). This, however, would serve little toward the purpose in view. The problem proposed is the derivation of formulas for the specifically defined solutions to which the symbols $J, Y$, and $H$ have by custom been assigned, and to this end a knowledge of the general solution of the equation in question does not suffice. It is over and above that essential that the solutions given be identified in order that the formulas obtained may be specific and involve no undetermined or arbitrary elements.

It was shown in $\$ 9$ how, with the use of the relations (47), the formulas for any solution specified in terms of its values at $x=0$ may be obtained from the formulas explicitly given. The mode of procedure there in question is, however, of no avail for the case in hand, for the values taken by the solutions (55) at $x=0$, far from being available for use in this way, are precisely among the quantities which it is proposed to derive. The method, therefore, which will be adopted to resolve the difficulty is distinct from any of those of the preceding sections. It will be clear in view of these remarks that the discussion which follows may naturally be expected to consist of two essentially distinct parts, and this division will in fact be readily observable. In the first part the immediate problem is the identification of the solutions (55) through the medium of certain of their elementary properties, and following this identification the later part of the discussion will be concerned with the derivation of the desired formulas from the general results of $\$ 9$.

12. The identification of the solutions. In virtue of the identical relation (56) it is obviously sufficient to identify any two of the solutions (55). The solutions to be chosen for this purpose will be those of the first and third kinds. The identification will be made in terms of the solutions $u_{1}(x)$ and $u_{2}(x)$ of equation (54) which are determined by the specifications of $\$ 9$. Inasmuch as these solutions are linearly independent there exists, of course, a pair of identical relations

(a)

$$
\begin{aligned}
J_{\rho}\left(\rho e^{x}\right) & =A_{1} u_{1}(x)+A_{2} u_{2}(x), \\
H_{\rho}^{(1)}\left(\rho e^{x}\right) & =B_{1} u_{1}(x)+B_{2} u_{2}(x),
\end{aligned}
$$


in which the coefficients $A_{i}, B_{i}$ are free from $x$ but are not necessarily independent of $\rho$. An identification of the solutions on the left of the equalities (58) will be effected through a determination of the coefficients on the right.

Consider to begin with the first of the relations (58). For negative values of $x$ the corresponding value of $\xi$ lies on the negative axis of imaginaries, i.e., in the half-plane $\Xi_{1}$. With the use of the relations (48a), therefore, the identity in question may be written

$$
J_{\rho}(\rho \operatorname{sech} \alpha) \equiv\left\{A_{1} a_{11}^{(1)}+A_{2} a_{21}^{(1)}\right\} u_{11}(x)+\left\{A_{1} a_{12}^{(1)}+A_{2} a_{22}^{(1)}\right\} u_{12}(x)
$$

Now when $|x|$ is large the asymptotic forms (41c) may be substituted in the right hand member of (59). As a result it becomes evident that the relation

$$
A_{1} a_{11}^{(1)}+A_{2} a_{21}^{(1)}=0
$$

must obtain, for in the alternative the first term on the right of the identity (59) would become infinite as $x \rightarrow-\infty$, whereas each remaining term of the identity approaches zero. Hence the relation at hand leads to the formula

$$
J_{\rho}(\rho \operatorname{sech} \alpha) \sim\left\{A_{1} a_{12}^{(1)}+A_{2} a_{22}^{(1)}\right\} \frac{e^{-\rho(\alpha-\tanh \alpha)}}{\rho^{1 / 6}(i \tanh \alpha)^{1 / 2}}\left\{1+\sum_{n=1}^{\infty} \frac{E_{n}(x, \rho)}{\rho^{n}}\right\} .
$$

Let each member of this formula be divided by the quantity $(\rho \operatorname{sech} \alpha)^{p}$. The limiting form of the relation which results, as sech $\alpha \rightarrow 0$, i.e., as $x \rightarrow-\infty$, is found then in virtue of the formula (45) to be

$$
\frac{1}{2^{\rho} \Gamma(\rho+1)}=\left\{A_{1} a_{12}^{(1)}+A_{2} a_{22}^{(1)}\right\} \frac{e^{\rho}}{\rho^{1 / 6} i^{1 / 2} \rho^{\rho} 2^{\rho}}\left\{1+\sum_{n=1}^{\infty} \frac{E_{n}(\rho)}{\rho^{n}}\right\} .
$$

It follows with the use of the familiar Stirling's formula,

$$
\Gamma(\rho+1)=\rho^{\rho} e^{-\rho}(2 \pi \rho)^{1 / 2}\left\{1+\sum_{n=1}^{\infty} \frac{E_{n}(\rho)}{\rho^{n}}\right\}^{*},
$$

that

$$
A_{1} a_{12}^{(1)}+A_{2} a_{22}^{(1)}=\left(\frac{i}{2 \pi}\right)^{1 / 2} \rho^{-1 / 3}\left\{1+\sum_{n=1}^{\infty} \frac{E_{n}(\rho)}{\rho^{n}}\right\}
$$

* Cf. Bromwich, An Introduction to the Theory of Infinite Series, 2d edition, London, 1926, p. 330 
The values of the coefficients $a_{j i}{ }^{(1)}$ are given by the formulas (53b). With these values substituted into the equations (60a) and (60b) there results an algebraic system which is easily solved and which gives the values

$$
\begin{aligned}
& A_{1}=\frac{1}{3^{1 / 2} \rho^{1 / 3}}\left\{1+\sum_{n=1}^{\infty} \frac{E_{n}(\rho)}{\rho^{n}}\right\}, \\
& A_{2}=\frac{1}{3^{1 / 2} \rho^{1 / 3}}\left\{1+\sum_{n=1}^{\infty} \frac{E_{n}(\rho)}{\rho^{n}}\right\} .
\end{aligned}
$$

The explicit form of the relations (58a) and (59) is thus found to be

(a) $J_{\rho}\left(\rho e^{x}\right)=\frac{1}{3^{1 / 2} \rho^{1 / 3}}\left\{u_{1}(x)\left[1+\sum_{n=1}^{\infty} \frac{E_{n}(\rho)}{\rho^{n}}\right]+u_{2}(x)\left[1+\sum_{n=1}^{\infty} \frac{E_{n}(\rho)}{\rho^{n}}\right]\right\}$,

(b) $J_{\rho}(\rho \operatorname{sech} \alpha) \sim\left(\frac{i}{2 \pi}\right)^{1 / 2} \rho^{-1 / 3} u_{12}(x)\left[1+\sum_{n=1}^{\infty} \frac{E_{n}(\rho)}{\rho^{n}}\right], \quad$ for $x<0$,

and the identification of the first of the solutions (55) is thus accomplished.

The principle thus employed in the derivation of formulas (61) may be used also to determine the coefficients in the identity $(58 \mathrm{~b})$. The details, however, are somewhat different. For positive values of $x$ the corresponding value $\xi$ lies on the positive axis of reals, namely, in the half-plane $\Xi_{0}$, and hence the relation (58b) may be written in the form

$$
H_{\rho}^{(1)}(\rho \sec \beta) \equiv\left\{B_{1} a_{11}^{(0)}+B_{2} a_{21}^{(0)}\right\} u_{01}(x)+\left\{B_{1} a_{12}^{(0)}+B_{2} a_{22}^{(0)}\right\} u_{02}(x) .
$$

Let this relation be multiplied, now, by the quantity

$$
\rho^{1 / 6}(\tan \beta)^{1 / 2} e^{-i \rho(\tan \beta-\beta)},
$$

and let the asymptotic forms (13) and (41c) be substituted respectively in the left and right hand members. The relation which thus results is found to be

$$
\begin{aligned}
& \left(\frac{2 \sin \beta}{\pi i}\right)^{1 / 2} \rho^{-1 / 3} e^{-\rho i(\pi / 2-\beta+\tan \beta-\sec \beta)}\left\{1+\sum_{n=1}^{\infty} \frac{E_{n}(\rho)}{(\rho \sec \beta)^{n}}\right\} \\
& \sim\left\{B_{1} a_{11}^{(0)}+B_{2} a_{21}^{(0)}\right\}\left\{1+\sum_{n=1}^{\infty} \frac{E_{n 1}(\rho)}{\rho^{n}}+\frac{E_{n 2}}{\xi^{n}}\right\} \\
& +\left\{B_{1} a_{12}^{(0)}+B_{2} a_{22}^{(0)}\right\} e^{-2 i \rho(\tan \beta-\beta)}\left\{1+\sum_{n=1}^{\infty} \frac{E_{n 3}(\rho)}{\rho^{n}}+\frac{E_{n 4}}{\xi^{n}}\right\}
\end{aligned}
$$


Now the coefficient of the final term in this relation must evidently be zero, for in the alternative this term would remain oscillatory as $\beta \rightarrow \pi / 2$, i.e., as $x \rightarrow \infty$, whereas the remaining terms approach definite and easily determined limits. Because of this fact and by means of an evaluation of the limits in question, it is found then that

$$
\begin{aligned}
& B_{1} a_{11}^{(0)}+B_{2} a_{21}^{(0)}=\left(\frac{2}{\pi i}\right)^{1 / 2} \rho^{-1 / 3}\left\{1+\sum_{n=1}^{\infty} \frac{E_{n}(\rho)}{\rho^{n}}\right\}, \\
& B_{1} a_{12}^{(0)}+B_{2} a_{22}^{(0)}=0 .
\end{aligned}
$$

This system of equations, with the values $a_{j i}{ }^{\left({ }^{0}\right)}$ given by the formulas (53b), is readily solved and gives the values

$$
\begin{aligned}
& B_{1}=\frac{2}{3^{1 / 2} \rho^{1 / 3}} e^{-\pi i / 3}\left\{1+\sum_{n=1}^{\infty} \frac{E_{n}(\rho)}{\rho^{n}}\right\}, \\
& B_{2}=\frac{2}{3^{1 / 2} \rho^{1 / 3}} e^{\pi i / 3}\left\{1+\sum_{n=1}^{\infty} \frac{E_{n}(\rho)}{\rho^{n}}\right\} .
\end{aligned}
$$

The explicit form of the relation (58b) is thus found to be

(a) $H_{\rho}^{(1)}(\rho \sec \beta) \sim\left(\frac{2}{\pi i}\right)^{1 / 2} \rho^{-1 / 3} u_{01}(x)\left[1+\sum_{n=1}^{\infty} \frac{E_{n}(\rho)}{\rho^{n}}\right]$, for $x>0$,

(b) $H_{\rho}^{(1)}\left(\rho e^{x}\right)=\frac{2}{3^{1 / 2} \rho^{1 / 3}}\left\{u_{1}(x) e^{-\pi i / 3}\left[1+\sum_{n=1}^{\infty} \frac{E_{n}(\rho)}{\rho^{n}}\right]\right.$

$$
\left.+u_{2}(x) e^{\pi i / 3}\left[1+\sum_{n=1}^{\infty} \frac{E_{n}(\rho)}{\rho^{n}}\right]\right\} \text {, for all } x,
$$

and this completes the identification of the solutions (55).

13. The asymptotic formulas when $|\xi|$ is large. When the quantity $\xi$ is large in absolute value the asymptotic forms for the various solutions $u$ are obtainable from the formulas (41c) and (53), by substituting in them the values given by the several relations (57). To facilitate this substitution it may be noted that since $1 / \xi=\phi^{-1}(x) / \rho$, while for the case in point $\sigma_{1}=1$, any product of the type

$$
\left\{1+\sum_{n=1}^{\infty} \frac{E_{n 1}(x, \rho)}{\rho^{n \sigma_{1}}}+\frac{E_{n 2}}{\xi^{n}}\right\}\left\{1+\sum_{n=1}^{\infty} \frac{E_{n}(\rho)}{\rho^{n}}\right\}
$$

is expressible in the form

$$
\left\{1+\sum_{n=1}^{\infty} \frac{P_{n}\left(\Phi^{-1}(x)\right)}{\rho^{n}}\right\}
$$


the symbol $P_{n}$ representing a polynomial of the degree $n$ in the argument shown, with coefficients which are bounded functions of $x$ and $\rho$. The symbol $P_{n}$ will be used in the following as a generic designation for such a polynomial function. As with the symbol $E$, so also with the symbol $P_{n}$, two functions similarly designated in different formulas are not to be considered as in general the same.

For positive values of $x$ the substitution of the form (41c) into the relation (62a) yields the asymptotic formula for the function $H_{\rho}^{(1)}$. The corresponding formula for the function $J_{\rho}$ is similarly obtained by the substitution of the forms (53) into the relation (61a), and the two formulas thus derived may be made to yield also that for the function $Y_{\rho}$ in virtue of the identity (56). Certain simple and obvious manipulations reduce the formulas in question to the forms

$$
\begin{aligned}
H_{\rho}^{(1)}(\rho \sec \beta)= & \left(\frac{2}{\pi \rho \tan \beta}\right)^{1 / 2} e^{i \rho(\tan \beta-\beta)-\pi i / 4}\left[1+\sum_{n=1}^{\infty} \frac{P_{n}\left(\frac{1}{\tan \beta-\beta}\right)}{\rho^{n}}\right], \\
J_{\rho}(\rho \sec \beta)= & \left(\frac{2}{\pi \rho \tan \beta}\right)^{1 / 2}\left[\cos \left(\rho \tan \beta-\rho \beta-\frac{\pi}{4}\right)\right. \\
& \cdot\left[1+\sum_{n=1}^{\infty} \frac{P_{n}\left(\frac{1}{\tan \beta-\beta}\right)}{\rho^{n}}\right] \\
& \left.+\sin \left(\rho \tan \beta-\rho \beta-\frac{\pi}{4}\right) \sum_{n=1}^{\infty} \frac{P_{n}\left(\frac{1}{\tan \beta-\beta}\right)}{\rho^{n}}\right], \\
Y_{\rho}(\rho \sec \beta)= & \left(\frac{2}{\pi \rho \tan \beta}\right)^{1 / 2}\left[\sin \left(\rho \tan \beta-\rho \beta-\frac{\pi}{4}\right)\right. \\
& {\left[1+\sum_{n=1}^{\infty} \frac{\left.P_{n}\left(\frac{1}{\tan \beta-\beta}\right)\right]}{\rho^{n}}\right] } \\
& \left.+\cos \left(\rho \tan \beta-\rho \beta-\frac{\pi}{4}\right) \sum_{n=1}^{\infty} \frac{P_{n}\left(\frac{1}{\tan \beta-\beta}\right)}{\rho^{n}}\right] .
\end{aligned}
$$

These relations are (to the extent to which the computation is explicit) essentially those by which the functions represented are customarily described [W, pp. 244, 245]. 
For negative values of $x$ such that $|\xi|$ is large the procedure is similar, being based now, however, upon the formulas (61b) and (62b). Inasmuch as any term involving a factor $e^{-\rho[\alpha-\tanh \alpha]}$ is asymptotically of negligible magnitude in the presence of any term involving a factor $\epsilon^{\rho[\alpha-\tanh \alpha]}$, the asymptotic formulas which are obtained in the manner indicated are found to be expressible in the form

$$
\begin{aligned}
J_{\rho}(\rho \operatorname{sech} \alpha) & \sim \frac{e^{-\rho(\alpha-\tanh \alpha)}}{(2 \pi \rho \tanh \alpha)^{1 / 2}}\left[1+\sum_{n=1}^{\infty} \frac{P_{n}\left(\frac{1}{\alpha-\tanh \alpha}\right)}{\rho^{n}}\right] \\
H_{\rho}^{(1)}(\rho \operatorname{sech} \alpha) & \sim \frac{-i e^{\rho(\alpha-\tanh \alpha)}}{\left(\frac{\pi}{2} \rho \tanh \alpha\right)^{1 / 2}}\left[1+\sum_{n=1}^{\infty} \frac{P_{n}\left(\frac{1}{\alpha-\tanh \alpha}\right)}{\rho^{n}}\right], \\
Y_{\rho}(\rho \operatorname{sech} \alpha) & \sim \frac{-e^{\rho(\alpha-\tanh \alpha)}}{\left(\frac{\pi}{2} \rho \tanh \alpha\right)^{1 / 2}}\left[1+\sum_{n=1}^{\infty} \frac{P_{n}\left(\frac{1}{\alpha-\tanh \alpha}\right)}{\rho^{n}}\right]
\end{aligned}
$$

These relations again are essentially those which are familiar for the description of the functions in question and for the range of argument considered [W, p. 243].

14. The asymptotic forms for intermediate values of $\xi$. For values of $x$ such that $|\xi|$ is bounded, i.e., such that $\xi$ is either of moderate magnitude or small, the appropriate formulas for the description of the solutions $u_{j}(x), j=1,2$, are those embodied in the relations (50). With these values substituted it is found that the formulas (61a) and (62b) lead to the forms

$$
\begin{aligned}
J_{\rho}\left(\rho e^{x}\right) & =\frac{\Psi(x) \xi^{1 / 3}}{3^{1 / 2} \rho^{1 / 3}}\left\{J_{-1 / 3}(\xi)+J_{1 / 3}(\xi)\right\}+\frac{E(x, \rho)}{\rho^{4 / 3}} \\
H_{\rho}^{(1)}\left(\rho e^{x}\right) & =\frac{2 \Psi(x) \xi^{1 / 3}}{3^{1 / 2} \rho^{1 / 3}}\left\{e^{-\pi i / 3} J_{-1 / 3}(\xi)+e^{\pi i / 3} J_{1 / 3}(\xi)\right\}+\frac{E(x, \rho)}{\rho^{4 / 3}}, \\
Y_{\rho}\left(\rho e^{x}\right) & =\frac{-\Psi(x) \xi^{1 / 3}}{\rho^{1 / 3}}\left\{J_{-1 / 3}(\xi)-J_{1 / 3}(\xi)\right\}+\frac{E(x, \rho)}{\rho^{4 / 3}}, \text { for }|\xi| \leqq N .
\end{aligned}
$$

Let the attention be given first to those values of $x$ which are positive and such that the magnitude of $\xi$ is moderate. In virtue of the values $(57 \mathrm{~b})$ the relations (65) become in this case the formulas 
(a) $\quad J_{\rho}(\rho \sec \beta)=\left(\frac{\tan \beta-\beta}{3 \tan \beta}\right)^{1 / 2}\left\{J_{-1 / 3}(\rho \tan \beta-\rho \beta)+J_{1 / 3}(\rho \tan \beta-\rho \beta)\right\}$

$$
+\frac{E(x, \rho)}{\rho^{4 / 3}}
$$

(b) $H_{\rho}^{(1)}(\rho \sec \beta)=2\left(\frac{\tan \beta-\beta}{3 \tan \beta}\right)^{1 / 2}\left\{e^{-\pi i / 3} J_{-1 / 3}(\rho \tan \beta-\rho \beta)\right.$

$$
\left.+e^{\pi i / 3} J_{1 / 3}(\rho \tan \beta-\rho \beta)\right\}+\frac{E(x, \rho)}{\rho^{4 / 3}},
$$

(c) $\quad Y_{\rho}(\rho \sec \beta)=-\left(\frac{\tan \beta-\beta}{\tan \beta}\right)^{1 / 2}\left\{J_{-1 / 3}(\rho \tan \beta-\rho \beta)\right.$

$$
\left.-J_{1 / 3}(\rho \tan \beta-\rho \beta)\right\}+\frac{E(x, \rho)}{\rho^{4 / 3}} .
$$

These forms it would seem have not been heretofore given, and a word of critique may, therefore, not be inappropriate. Formulas for the functions represented by the relations (66), and for the range of argument at present under consideration, have, of course, been previously known. Their derivation is due to Watson [W, p. 249]. Incidentally the procedure by which they were obtained is described by its author as "a method which is theoretically simple (though actually it is very laborious)."

For purposes of comparison just one of Watson's formulas may here be noted, namely,

$$
\begin{aligned}
J_{\rho}(\rho \sec \beta) & =\frac{1}{3} \tan \beta \cos \left(\rho\left[\tan \beta-\frac{1}{3} \tan ^{3} \beta-\beta\right]\right)\left\{J_{-1 / 3}\left(\frac{1}{3} \rho \tan ^{3} \beta\right)\right. \\
+J_{1 / 3} & \left.\left(\frac{1}{3} \rho \tan ^{3} \beta\right)\right\}+\frac{1}{3^{1 / 2}} \tan \beta \sin \left(\rho\left[\tan \beta-\frac{1}{3} \tan ^{3} \beta-\beta\right]\right) \\
& \left\{\left\{J_{-1 / 3}\left(\frac{1}{3} \rho \tan ^{3} \beta\right)-J_{-1 / 3}\left(\frac{1}{3} \rho \tan ^{3} \beta\right)\right\}+\frac{24 \theta_{2}}{\rho}, \quad\left|\theta_{2}\right|<1 .\right.
\end{aligned}
$$

The complexity of this formula need hardly be remarked upon. It is a feature also of Watson's corresponding formula for the function $Y_{\rho}$. It will be evident that in comparison the formulas (66) are very pronouncedly simpler. Aside from this it should be observed that the formulas (66) are explicit to terms of the order $O\left(\rho^{-4 / 3}\right)$ while the degree of explicitness of foimula (67) and of its companion formula for $Y_{\rho}$ extends only to the order $O\left(\rho^{-1}\right)$. 
There is, of course, no conflict between the formulas (66) and those of which (67) is representative. The formulas in question, it will be recalled, are all designed for use when $\xi$ is of moderate magnitude. This is readily translated into the condition that the value of $\beta$ be of the order $O\left(\rho^{-1 / 3}\right)$, and for such values of $\beta$ the quantities $(\tan \beta-\beta)$ and $\frac{1}{3} \tan ^{3} \beta$ differ by an amount which is of the order $O\left(\rho^{-5 / 3}\right)$. With the use of this fact, the agreement of the formulas (66) with those represented by (67) may be established to the extent to which both sets of formulas are explicit.

Let the attention be turned now to the negative values of $x$ for which $|\xi|$ is moderate. In this case $\xi$ is pure imaginary with $\arg \xi=3 \pi / 2$, and it is accordingly convenient to utilize the familiar Bessel functions $I_{ \pm 1 / 3}$, and $K_{1 / 3}$, which may be taken as defined by the relations [W, pp. 77, 78]

$$
\begin{aligned}
J_{\mp 1 / 3}(z) & =\mp i I_{\mp 1 / 3}\left(z e^{-3 \pi i / 2}\right), \\
K_{1 / 3}(z) & =\frac{3^{1 / 2}}{\pi}\left\{I_{-1 / 3}(z)-I_{1 / 3}(z)\right\} .
\end{aligned}
$$

The substitution of these functions together with the values $(57 \mathrm{c})$ into the formulas (65) gives to the latter the forms

$$
\begin{aligned}
J_{\rho}(\rho \operatorname{sech} \alpha)= & \frac{1}{\pi}\left(\frac{\alpha-\tanh \alpha}{\tanh \alpha}\right)^{1 / 2} K_{1 / 3}(\rho \alpha-\rho \tanh \alpha)+\frac{E(x, \rho)}{\rho^{4 / 3}}, \\
H_{\rho}^{(1)}(\rho \operatorname{sech} \alpha)= & 2\left(\frac{\alpha-\tanh \alpha}{3 \tanh \alpha}\right)^{1 / 2}\left\{e^{-\pi i / 3} I_{-1 / 3}(\rho \alpha-\rho \tanh \alpha)\right. \\
& \left.-e^{\pi i / 3} I_{1 / 3}(\rho \alpha-\rho \tanh \alpha)\right\}+\frac{E(x, \rho)}{\rho^{4 / 3}} \\
Y_{\rho}(\rho \operatorname{sech} \alpha)= & -\left(\frac{\alpha-\tanh \alpha}{\tanh \alpha}\right)^{1 / 2}\left\{I_{-1 / 3}(\rho \alpha-\rho \tanh \alpha)\right. \\
& \left.+I_{1 / 3}(\rho \alpha-\rho \tanh \alpha)\right\}+\frac{E(x, \rho)}{\rho^{4 / 3}} .
\end{aligned}
$$

These formulas as well as those in (66) appear to be new. Watson has given for the function $J_{\rho}$ and the range of argument under consideration the formula $[\mathrm{W}$, p. 250]

$$
\begin{aligned}
J_{\rho}(\rho \operatorname{sech} \alpha)= & \frac{\tanh \alpha}{\pi 3^{1 / 2}} e^{\rho\left(\tanh \alpha-(1 / 3) \tanh ^{3} \alpha-\alpha\right)} K_{1 / 3}\left(\frac{1}{3} \rho \tanh ^{3} \alpha\right) \\
& +\frac{3 \theta_{1}}{\rho} e^{\rho(\tanh \alpha-\alpha)},\left|\theta_{1}\right|<1 .
\end{aligned}
$$


In comparison with this the first of the formulas (68) has an evident advantage of simplicity. Its additional explicitness to a term in $\rho^{-4 / 3}$ may be contrasted with the explicitness of (69) which extends only to the order of $\rho^{-1}$. That agreement exists between the formula (69) and the corresponding formula of the set (68) may be established on the basis of considerations entirely similar to those specifically pointed out in the discussion of the formulas (66) above.

15. The formulas when argument and order are nearly equal. The values of $x$ for which $|\xi|$ is small, i.e., $\xi=o(1)$, are by formula (5a) the values characteristic of the relation $x=o\left(\rho^{-2 / 3}\right)$. It is convenient for this range of values to introduce the quantities $z$ and $\epsilon$ by means of the relations

$$
z=\rho e^{x}, \rho=z(1-\epsilon) .
$$

It is found then that $\epsilon z=o\left(\rho^{-1 / 3}\right)$. Moreover, in terms of these variables $z, \epsilon$ the relations (57a) are readily shown to be expressible in the manner

$$
\begin{aligned}
\phi(x) & =(2 \epsilon)^{1 / 2}\left\{1+\frac{3 \epsilon z}{4 z}+\cdots\right\}, \\
\xi & =\frac{(2 \epsilon z)^{3 / 2}}{3 z^{1 / 2}}\left\{1+\frac{\epsilon z}{20 z}+\cdots\right\}, \\
\frac{\Psi(x)}{\rho^{1 / 3}} & =\frac{1}{3^{1 / 6} z^{1 / 3}}\left\{1+\frac{2 \epsilon z}{15 z}+\cdots\right\} .
\end{aligned}
$$

Since the value of $\xi$ is small the Bessel functions involved in the right hand members of the formulas (65) are essentially evaluated by the initial terms of their power series expansions, i.e.,

$$
\begin{aligned}
\xi^{1 / 3} J_{-1 / 3}(\xi) & =\frac{2^{1 / 3}}{\Gamma\left(\frac{2}{3}\right)}-\frac{\xi^{2}}{2^{5 / 3} \Gamma\left(\frac{5}{3}\right)}+\cdots \\
\xi^{1 / 3} J_{1 / 3}(\xi) & =\frac{\xi^{2 / 3}}{2^{1 / 3} \Gamma\left(\frac{4}{3}\right)}+\cdots
\end{aligned}
$$

These evaluations may be expressed in terms of the quantities $z$ and $\epsilon$ in virtue of the relations (70). With the use of the familiar formula

$$
3^{1 / 2} \Gamma\left(\frac{1}{3}\right) \Gamma\left(\frac{2}{3}\right)=2 \pi,
$$


it is found then that

(71)

$$
\xi^{1 / 3} J_{-1 / 3}(\xi)=\frac{3^{1 / 2} \Gamma\left(\frac{1}{3}\right)}{2^{2 / 3} \pi}-\frac{3^{1 / 2} \Gamma\left(\frac{4}{3}\right)(\epsilon z)^{3}}{2^{2 / 3} \pi z}+\cdots
$$

$$
\xi^{1 / 3} J_{1 / 3}(\xi)=\frac{3^{5 / 6} \Gamma\left(\frac{2}{3}\right)}{2^{1 / 3} \pi z^{1 / 3}}+\cdots
$$

The values (70) and (71) may now be substituted into the relations (65) and give to the latter the forms

$$
\begin{aligned}
J_{\rho}(z)= & \frac{1}{2^{2 / 3} 3^{1 / 6} \pi}\left[\frac{\Gamma\left(\frac{1}{3}\right)}{z^{1 / 3}}+\frac{6^{1 / 3} \Gamma\left(\frac{2}{3}\right) \epsilon z}{z^{2 / 3}}\right. \\
& \left.-\frac{6 \Gamma\left(\frac{4}{3}\right)\left\{\frac{(\epsilon z)^{3}}{6}-\frac{\epsilon z}{15}\right\}}{z^{4 / 3}}+\cdots\right]+\frac{E(x, \rho)}{\rho^{4 / 3}},
\end{aligned}
$$

$$
\begin{aligned}
H_{\rho}^{(1)}(z)= & \frac{2^{1 / 3}}{3^{1 / 6} \pi}\left[\frac{\Gamma\left(\frac{1}{3}\right) e^{-\pi i / 3}}{z^{1 / 3}}+\frac{6^{1 / 3} \Gamma\left(\frac{2}{3}\right) e^{\pi i / 3} \epsilon z}{z^{2 / 3}}\right. \\
& \left.-\frac{6 \Gamma\left(\frac{4}{3}\right) e^{-\pi i / 3}\left\{\frac{(\epsilon z)^{3}}{6}-\frac{\epsilon z}{15}\right\}}{z^{4 / 3}}+\cdots\right]+\frac{E(x, \rho)}{\rho^{4 / 3}}, \\
Y_{\rho}(z)= & \frac{-3^{1 / 3}}{2^{2 / 3} \pi}\left[\frac{\Gamma\left(\frac{1}{3}\right)}{z^{1 / 3}}-\frac{6^{1 / 3} \Gamma\left(\frac{2}{3}\right) \epsilon z}{z^{2 / 3}}\right. \\
& \left.-\frac{6 \Gamma\left(\frac{4}{3}\right)\left\{\frac{(\epsilon z)^{3}}{6}-\frac{\epsilon z}{15}\right\}}{z^{4 / 3}}+\cdots\right]+\frac{E(x, \rho)}{\rho^{4 / 3}} .
\end{aligned}
$$

To the extent to which these formulas are explicit they are precisely those which are customarily given for the description of the functions in question when the argument $z$ is nearly equal to the order $\rho$ [W, p. 247]. 
Lastly the value of $\epsilon$ may be set equal to zero, i.e., $z$ may be set equal to $\rho$. The resultant reduction in the relations (72) yields then the familiar formulas [W, p. 232]

(73)

$$
\begin{gathered}
J_{\rho}(\rho)=\frac{\Gamma\left(\frac{1}{3}\right)}{2^{2 / 3} 3^{1 / 6} \pi \rho^{1 / 3}}+O\left(\rho^{-4 / 3}\right), \\
H_{\rho}^{(1)}(\rho)=\frac{2^{1 / 3} \Gamma\left(\frac{1}{3}\right)}{3^{1 / 6} \pi \rho^{1 / 3}}+O\left(\rho^{-4 / 3}\right), Y_{\rho}(\rho)=\frac{-3^{1 / 3} \Gamma\left(\frac{1}{3}\right)}{2^{2 / 3} \pi \rho^{1 / 3}}+O\left(\rho^{-4 / 3}\right),
\end{gathered}
$$

and with this result the discussion will be concluded.

UNIVERSITY OF WISCONSIN, Madison, Wis. 